\title{
Languages of Higher-Dimensional Automata
}

\author{
École Polytechnique, Palaiseau, France \\ Norwegian University of Science and Technology, Norway \\ University of Sheffield, UK \\ University of Warsaw, Poland
}

Uli Fahrenberg, Christian Johansen, Georg Struth, and Krzysztof Ziemiański

\begin{abstract}
We introduce languages of higher-dimensional automata (HDAs) and develop some of their properties. To this end, we define a new category of precubical sets, uniquely naturally isomorphic to the standard one, and introduce a notion of event consistency. HDAs are then finite, labeled, event-consistent precubical sets with distinguished subsets of initial and accepting cells. Their languages are sets of interval orders closed under subsumption; as a major technical step we expose a bijection between interval orders and a subclass of HDAs. We show that any finite subsumption-closed set of interval orders is the language of an HDA, that languages of HDAs are closed under binary unions and parallel composition, and that bisimilarity implies language equivalence.

MSC 2020: Primary 68Q70, 68Q85
\end{abstract}

Keywords: Higher-dimensional automaton; concurrency theory; pomset; directed topology

\section{Introduction}

Higher-dimensional automata (HDAs) are a formalism for modeling and reasoning about behaviors of concurrent systems, introduced by Pratt [34] and van Glabbeek [40]. Like Petri nets [32], event structures [30], configuration structures [45, 46], asynchronous transition systems [2, 39], and similar approaches [24, 35, 37, 44], they form a model of non-interleaving concurrency as they differentiate between interleaving and "truly" concurrent computations, i.e., $a \| b \neq a . b+b . a$ (using CCS notation [29]). Van Glabbeek [42] has shown that HDAs generalize "the main models of concurrency proposed in the literature", including those mentioned above.

HDAs extend finite automata with additional structure that distinguishes interleavings from concurrency. As an example, Figure 1 shows Petri net and HDA models for a system with two events, labeled $a$ and $b$. The Petri net and HDA on the left model the (mutually exclusive) interleaving of $a$ and $b$ as either $a . b$ or $b . a$; those on the right model concurrent execution of $a$ and $b$. In the HDA, this independence is indicated by a filled-in square.
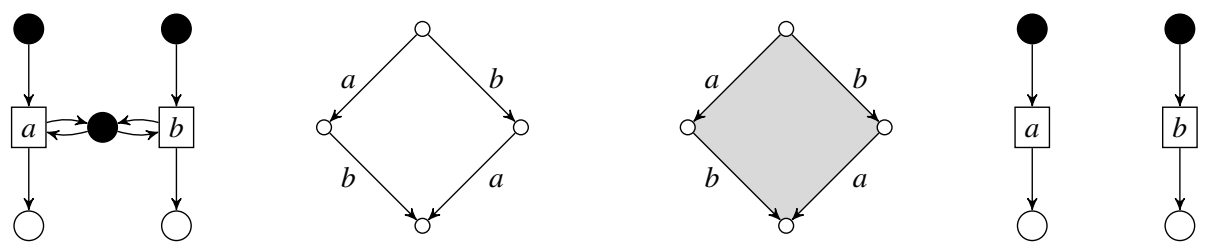

Figure 1. Petri net and HDA models distinguishing interleaving (left) from non-interleaving (right) concurrency. Left: Petri net and HDA models for $a . b+b . a$; right: HDA and Petri net models for $a \| b$. 


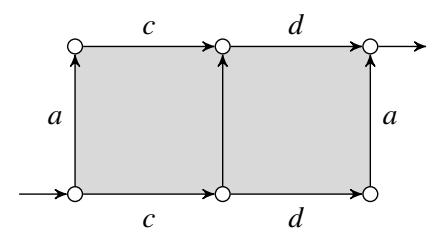

Figure 2. HDA which executes $a$ in parallel with c.d. Initial and accepting cells marked with incoming and outgoing arrows.

HDAs thus have states and transitions like finite automata, but may also contain squares, cubes, and higher-dimensional cubical structures. A square stands for the concurrent execution of two events; a cube for the concurrent execution of three events; and so on.

This paper is concerned with languages of HDAs. Like languages related to other formalisms for concurrency, these need to account for both the sequential and the concurrent nature of computations. Their elements will therefore be finite pomsets or partial words [49]. As an example, Figure 2 displays an HDA consisting of two squares, with three events labeled $a, c$, and $d$. Here the $a$-labeled event is executed concurrently to the sequence $c . d$, so that the language of this HDA will contain the pomset

$$
(c \stackrel{a}{\longrightarrow} d) .
$$

(It will contain other elements; but in a sense to be made precise below, they are all generated by this one pomset.)

Partial words and pomsets have been introduced by Winkowski [49] and have a long history as semantics for concurrent systems [33, 48]. The subclass of interval orders, introduced by Fishburn [15], has seen abundant attention in concurrency theory and distributed systems [8, 21, 22, 23, 26, $27,47,48]$. A pomset is an interval order precisely if it is $2+2$-free, that is, does not contain an induced subpomset of the form

$$
2+2=(\bullet \longrightarrow \bullet)
$$

We will show that languages of HDAs are sets of interval orders, and that any interval order may be generated by an HDA. For another example, the HDA in Figure 3 has a two-dimensional loop created by identifying the horizontal edges in the bottom-left and top-right of the automaton (together with their corresponding faces). Its language includes the infinite set

$$
\left\{(a \longrightarrow b \longrightarrow a),\left(\begin{array}{l}
a \longrightarrow b \longrightarrow a \longrightarrow b \longrightarrow a \\
\longrightarrow_{c} \longrightarrow d \longrightarrow a
\end{array}\right), \ldots\right\},
$$

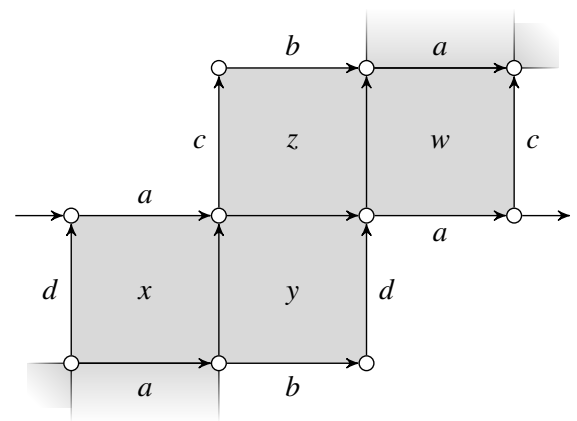

Figure 3. HDA which generates infinite set of pomsets (bottom left and top right edges identified). 


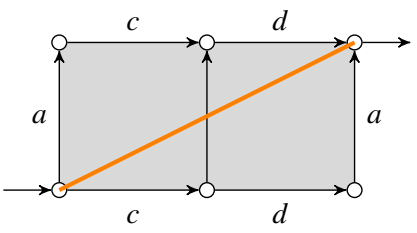

$(c \stackrel{a}{\longrightarrow} d)$

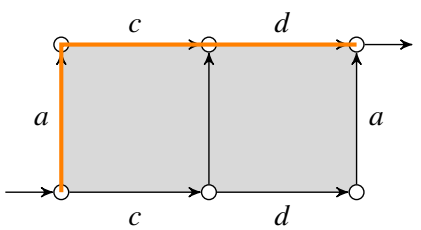

$(a \longrightarrow c \longrightarrow d)$

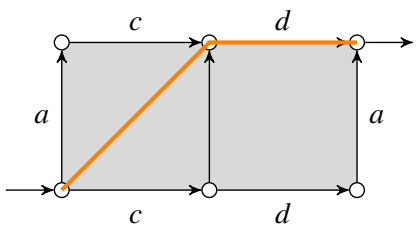

$\left(\begin{array}{l}a \longrightarrow d \\ c \longrightarrow d\end{array}\right)$

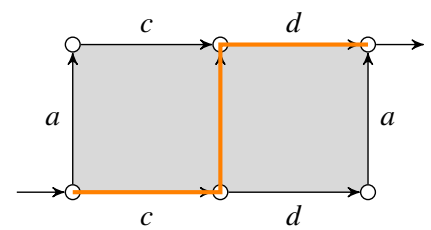

$(c \longrightarrow a \longrightarrow d)$

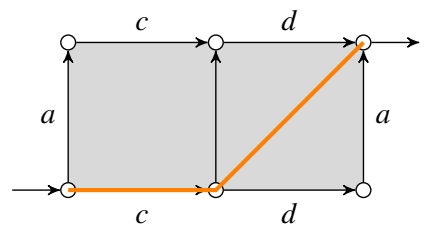

$\left(\begin{array}{l}a \\ c \rightarrow d\end{array}\right)$

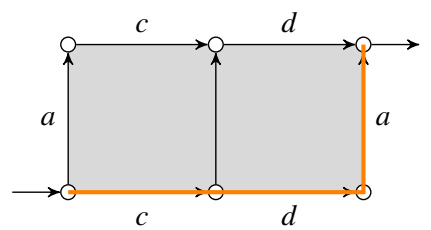

$(c \longrightarrow d \longrightarrow a)$

Figure 4. Directed paths in HDA of Figure 2 together with corresponding pomsets.

where the second pomset is obtained by traversing the squares $z, w, x$ and $y$ in that order. We will only be concerned with finite HDAs in this paper, yet as the above example shows, languages of finite HDAs may well be infinite.

A precursor to this work is van Glabbeek's [42], which introduces tracks in HDAs (there called paths) and then defines their observable content in terms of ST-traces. We have shown in [8] that there is a bijective correspondence between ST-traces and interval orders. Another precursor is Fajstrup et al.'s [13], where the authors define computations as directed paths through geometric cubical complexes. We introduce languages based on van Glabbeek's tracks and languages based on Fajstrup et al.'s directed paths, and show that they define the same objects.

Grabowski [18] has introduced a notion of smoothing for pomsets which is nowadays mostly called subsumption [14, 17]: a pomset $P$ subsumes a pomset $Q$ if $Q$ is at least as ordered as $P$. Sets of pomsets closed under subsumption are generally called weak $[14,18]$. We show that languages of HDAs are weak sets of interval orders.

Figure 4 exhibits six directed paths through the HDA $A$ of Figure 2 together with the corresponding pomsets. The language of $A$ consists precisely of these six pomsets; it is also the weak closure of the pomset in (1) corresponding to the first directed path displayed. The language of the HDA in Figure 3 is the weak closure of the infinite set in (2).

We finish the paper by showing that languages of HDAs are closed under binary union and parallel composition, and further that bisimilarity of HDAs [10, 42] implies language equivalence. A comprehensive treatment of regular operations on HDAs and their languages is left for future work.

We start this paper with an overview section which introduces the main concepts and results without going into too much technical detail. In order to properly define and develop languages of HDAs, we first introduce a new base category for precubical sets, identify a new subclass of event consistent precubical sets, and make clear the relationship between tracks and interval orders. This is also why we define HDAs only on page 13 .

We detail the main technical contributions of this paper at the end of the overview Section 2. Afterwards, we introduce precubical sets, event consistency, and HDAs in Section 3. Section 4 is concerned with pomsets with interfaces, their gluing composition, and representations of interval orders. The connection between interval orders and tracks in precubical sets is made in 


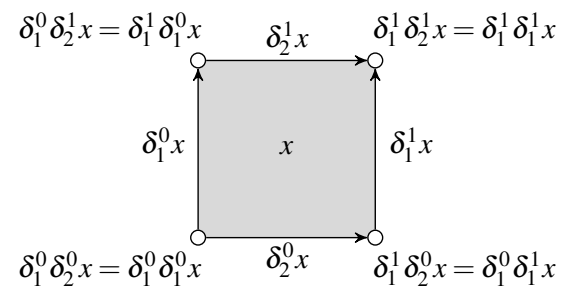

Figure 5. A square $x$ with its four elementary faces $\delta_{1}^{0} x, \delta_{1}^{1} x, \delta_{2}^{0} x, \delta_{2}^{1} x$ and four corners.

Section 5, and directed paths are introduced in Section 6. Section 7 concludes the paper by defining languages of HDAs and developing some basic properties.

\section{Overview}

HDAs are built on precubical sets [19, 38], a generalization of directed graphs to higher dimensions. To be precise, a precubical set consists of a graded set $X=\bigcup_{n \geq 0} X_{n}$ of $n$-cells together with elementary face maps $\delta_{i, n}^{v}: X_{n} \rightarrow X_{n-1}, i \in\{1, \ldots, n\}, v \in\{0,1\}$ that specify boundaries of $n$-cells. These are required to satisfy the precubical identities

$$
\delta_{i, n-1}^{v} \delta_{j, n}^{\mu}=\delta_{j-1, n-1}^{\mu} \delta_{i, n}^{v},
$$

for every $i<j \leq n$, which identify common elementary faces of elementary faces. Figure 5 shows an example of a 2-cell with all its faces; we will generally omit parentheses for elementary faces and the subscript $n$ and thus, for example, write $\delta_{1}^{0} x$ instead of $\delta_{1, n}^{0}(x)$.

A precubical set $X$ with $X_{1}=\emptyset$, hence $X_{i}=\emptyset$ for all $i \geq 1$, is simply a set (of 0-cells or points). A one-dimensional precubical set $X$, with $X_{2}=\emptyset$, is a directed graph. 1-cells are generally called edges, 2-cells, squares, and 3-cells, cubes. Modifying the standard setting [19], we introduce precubical sets as presheaves over a category of linearly ordered sets with suitable morphisms (Definition 1). From a technical point of view this does not matter, as our "large" category of precubical sets is uniquely isomorphic to the standard one (Proposition 9); yet it clarifies the relation between ordered sets, presimplicial sets and precubical sets. This simplifies later developments.

An HDA is a tuple $(X, I, F, \lambda)$ with $X$ a precubical set, $I, F \subseteq X$ subsets of initial and accepting cells, and $\lambda$ a labeling on $X$. This labeling is generated by a function $\lambda_{1}: X_{1} \rightarrow \Sigma$, into an alphabet $\Sigma$, which satisfies $\lambda_{1}\left(\delta_{1}^{0} x\right)=\lambda_{1}\left(\delta_{1}^{1} x\right)$ and $\lambda_{1}\left(\delta_{2}^{0} x\right)=\lambda_{1}\left(\delta_{2}^{1} x\right)$ for every $x \in X_{2}$; but we will extend it to a precubical morphism $\lambda: X \rightarrow ! \Sigma$ into a special labeling object! $\Sigma$ (Definition 13).

One-dimensional HDAs are equivalent to ordinary finite automata, with 0-cells as states and 1 -cells as transitions. Two-dimensional HDAs are equivalent to asynchronous transition systems, with the 2-cells denoting independence of events.

Most formalisms for non-interleaving concurrency have a notion of events: unique occurrences of actions in space and time. HDAs, on the other hand, do not have a well-defined notion of event $[9,36]$. Going back to the example in Figure 1, we see that the Petri nets on each side of the figure have two events each, induced by their transitions and labeled $a$ and $b$, respectively. In the two HDAs on the other hand, every label appears twice, and there is no immediate conception of events. For the HDA on the right, we may deduce from the presence of the square, which indicates two events running concurrently, that there are indeed precisely two events in the system; but on the left, there might as well be four.

We make the notion of event identification precise in Definition 13 and identify a subclass of event consistent precubical sets: precubical sets $X$ that admit an equivalence relation $\sim$ on $X_{1}$ such that for all $x \in X_{2}, \delta_{1}^{0} x \sim \delta_{1}^{1} x, \delta_{2}^{0} x \sim \delta_{2}^{1} x$, and $\delta_{1}^{0} x \neq \delta_{2}^{0} x$ (Lemma 18). The equivalence 


$$
\left.\left(\begin{array}{r}
a \\
b \longrightarrow c
\end{array}\right) *{ }^{a} \begin{array}{l}
a \\
d
\end{array}\right)=\left(\begin{array}{c}
a \\
b \longrightarrow c \longrightarrow d
\end{array}\right)
$$

Figure 6. Two ipomsets and their gluing composition (interfaces marked with incoming and outgoing arrows).
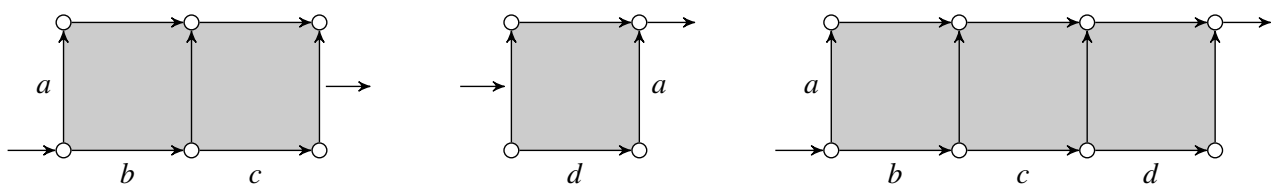

Figure 7. Three HDAs corresponding to ipomsets of Figure 6.

classes of the smallest such equivalence are called the universal events of $X$ : the largest possible identification of events which is consistent with the structure of the HDA.

In the example in Figure 1, the HDA on the left has four universal events, whereas the one on the right has two. (An example of a precubical set which is not event consistent is shown in Figure 10 on page 11.)

Any labeling factors uniquely through the universal events (Proposition 20), so that we could have written this paper only with unlabeled (but event consistent) HDAs in mind and then added labels as an afterthought, much in the spirit of [50]. For sake of readability we have refrained from doing so.

In Section 4 we recapitulate the notion of pomset with interfaces (ipomset) from [8]. An ipomset $\left(P,<_{P}, \lambda_{P}, S_{P}, T_{P}\right)$ consists of a labeled partial order $\left(P,<_{P}, \lambda_{P}\right)$ together with subsets $S_{P}, T_{P} \subseteq P$ of minimal and maximal elements which designate starting and terminating interfaces. Ipomsets may be glued along their interfaces: if $\left(Q,<_{Q}, \lambda_{Q}, S_{Q}, T_{Q}\right)$ is another ipomset such that $P \cap Q=$ $T_{P}=S_{Q}$, then $P * Q$ is the ipomset

$$
\left(P \cup Q,<_{P} \cup<_{Q} \cup\left(P \backslash T_{P}\right) \times\left(Q \backslash S_{Q}\right), \lambda_{P} \cup \lambda_{Q}, S_{P}, T_{Q}\right),
$$

with the order defined by those of $P$ and $Q$ together with imposing that every event not in $Q$ precedes every event not in $P$. Hence events in the overlap $P \cap Q$ are continued across the gluing composition; Figure 6 shows an example.

We extend ipomsets with an event order: a second strict order, denoted $-\rightarrow P$, which is required to be linear on $<_{P}$-antichains. This allows us to assign which interfaces are identified in gluing compositions and also establishes a close relation between interval-ordered ipomsets and a subclass of HDAs, see Definition 60. As an example, Figure 7 shows the three HDAs corresponding to the ipomsets of Figure 6; we show in Lemma 65 that gluing compositions of interval ipomsets correspond to pushouts of their induced HDAs.

Most papers in concurrency theory define pomsets as isomorphism classes of labeled partial orders. We find it convenient to work directly with labeled partial orders instead and consider properties up to isomorphism. As any isomorphic ipomsets are uniquely isomorphic (Lemma 34), the difference is without significance.

One central mathematical insight on which this paper is built is that both precubical sets and interval ipomsets can be obtained by gluing linear orders, i.e., precubical sets glued as presheaves, and interval ipomsets as gluing compositions of discrete ipomsets. An ipomset $\left(P,<_{P},-\rightarrow_{P}, \lambda_{P}, S_{P}, T_{P}\right)$ is discrete if $<_{P}$ is trivial, thus $\rightarrow_{P}$ is a linear order. We always think of $<_{P}$ as a precedence order; hence all events are concurrent in a discrete ipomset, and the event order $\rightarrow \rightarrow P$ is used as a book-keeping device. Seen as linear $\rightarrow \rightarrow P$-ordered sets, discrete ipomsets form our base category for precubical sets; as trivial $<_{P}$-ordered sets, we may glue them into interval orders. 


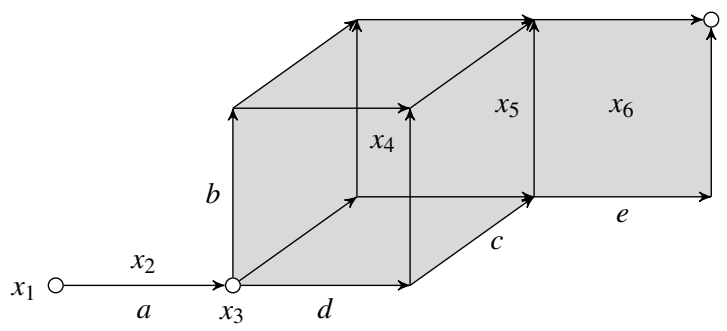

Figure 8. A track in a precubical set.

The subclass of precubical sets that correspond to interval ipomsets under this identification is comprised of tracks: sequences of cells connected at intermediate faces. This notion generalizes paths in finite automata to higher dimensions. Figure 8 shows an example, a track consisting of six cells $x_{1}, \ldots, x_{6}\left(x_{4}\right.$ is the central cube in the figure) with face relations

$$
x_{1} \triangleleft x_{2} \triangleright x_{3} \triangleleft^{3} x_{4} \triangleright^{2} x_{5} \triangleleft x_{6},
$$

where $x_{1} \triangleleft x_{2}$ denotes that $x_{1}$ is a lower face of $x_{2}$, and $x_{4} \triangleright^{2} x_{5}$ that $x_{5}$ is an upper face of an upper face of $x_{4}$ (in anticipation of notation introduced later on). We show in Section 5 how tracks give rise to interval ipomsets, but also how interval ipomsets can be converted into tracks.

In Section 6 we give a geometric interpretation of executions in HDAs, following [13] and subsequent related work [12, 51, 52]. Precubial sets may be realized geometrically as directed spaces [19], and executions of HDAs may then be seen as directed paths through their geometric realization. We introduce the interval arrangement of a directed path, which tracks the events that are active during different phases of the execution, and use this to define labels of directed paths.

In Section 7 we show that languages of HDAs defined by directed paths are the same as languages defined by tracks. We also see that languages of HDAs are weak sets of interval orders, and that any finite weak set of interval orders may be generated by an HDA.

In summary, the main contributions of this paper are as follows:

- New Definitions 1 and 10 of precubical sets as presheaves over a category $\square$. This has linearly ordered sets as objects, and the morphism are pairs $(f, \varepsilon)$ of a poset map $f$ and a function $\varepsilon$ which partitions elements not in the image of $f$ into two classes. This is similar to constructions in $[1,3]$; the standard base category $\square$ [19] of precubical sets is uniquely isomorphic to the skeleton of $\square$.

- The identification of a new subclass of event consistent precubical sets in Definitions 13 and 17 and the introduction of universal events for such precubical sets.

- The exposition of a bijection, in Definitions 55 and 60, between interval-ordered ipomsets and HDA tracks. The first of these definitions introduces the label of a track in an HDA $X$, which forms the basis on which we define track-based languages of HDAs; the second defines the track object $\square^{P}$ pertaining to an interval ipomset $P$. These notions unite in the important Proposition 89: $P$ is contained in the language of $X$ precisely if there is an HDA morphism from $\square^{P}$ into $X$.

- The notion of interval arrangement of a directed path through the geometric realization of an HDA and the subsequent Definition 77 of labels of directed paths.

- Definition 86 of the language of an HDA, the closure properties (under binary union and parallel composition) in Theorems 100 and 108, and Theorems 110 and 111 that bisimilarity implies language equivalence. We expect that together with Proposition 89 this may form the basis of a theory of regular pomset languages, but leave this for future work. 


\section{Precubical Sets and Higher Dimensional Automata}

In this section we introduce precubical sets and HDAs, but we start with order-theoretic definitions, mainly to fix notation. We will return to posets and posets with interfaces in the next section.

A poset is a pair $(P,<)$ consisting of a set $P$ and a strict partial order $<$ on $P$. We henceforth assume tacitly that the set $P$ is finite. For any alphabet, i.e., finite set $\Sigma$, a pomset is a triple $(P,<, \lambda)$ with $(P,<)$ a poset and $\lambda: P \rightarrow \Sigma$ the labeling of $P$. If the order is linear, i.e., a total relation in which $x=y, x<y$, or $y<x$ for all $x, y \in P$, then we will speak of linear posets and linear pomsets (and generally denote linear po(m)sets by $S, T, U$ instead of $P, Q, R$ ).

Elements $x, y \in P$ of a po(m)set $P$ are comparable if $x=y, x<y$, or $y<x$; otherwise they are incomparable, denoted $x \| y$. An element $x \in P$ is minimal if there exists no $y \in P$ with $y<x$; and maximal if there is no $y \in P$ with $x<y$.

A subset $Q \subseteq P$ of a po(m)set $P$ is an antichain if its elements are pairwise incomparable. A maximal antichain is one which is not a proper subset of any other antichain. The sets of minimal, respectively maximal elements of $P$ are both maximal antichains.

A function $f: P \rightarrow Q$ between posets $P, Q$ is a poset map a if $x<_{P}$ y implies $f(x)<_{Q} f(y)$ for all $x, y \in P$. By irreflexivity, $f$ is injective on comparable elements: if $x<_{P} y$ or $y<_{P} x$, then $f(x) \neq f(y)$. If $P$ is linearly ordered, then $f$ must be injective.

A function $f: P \rightarrow Q$ between pomsets $P, Q$ is a pomset map if it is a poset map that preserves the labeling, i.e., $\lambda_{Q} \circ f=\lambda_{P}$. Posets and poset maps form the category Pos, and pomsets and pomset maps form the category Poms. Isomorphism in these and all subsequent categories will be denoted $\cong$.

\subsection{Precube Categories}

Precubical sets are usually defined as presheaves over a small skeletal category $\square$, see Definition 2 below. We find it more convenient to work with a large version of $\square$, denoted $\square$ and defined below, which as objects has all linear posets.

Definition 1. The large precube category $\square$ consists of the following data:

- objects are linear posets $(S,-\rightarrow)$;

- morphisms $S \rightarrow T$ in $\bullet(S, T)$ are pairs $(f, \varepsilon)$, where $f: S \rightarrow T$ is a poset map and $\varepsilon: T \rightarrow$ $\left\{0,\ulcorner, 1\}\right.$ a function such that $f(S)=\varepsilon^{-1}(\ulcorner)$;

- the composition of morphisms $(f, \varepsilon): S \rightarrow T$ and $(g, \zeta): T \rightarrow U$ is $(g \circ f, \eta)$, where

$$
\eta(u)= \begin{cases}\varepsilon\left(g^{-1}(u)\right) & \text { for } u \in g(T), \\ \zeta(u) & \text { otherwise }\end{cases}
$$

The function $\varepsilon$ distinguishes events that have not yet started (labelled by 0 ) from those that have finished (labelled by 1 ) and those that are executing (labelled by $\ulcorner$ ). This notation is inspired by Chu spaces [35]; see also [9] for the relation between HDAs and Chu spaces. For every morphism $(f, \varepsilon)$, the isomorphism $f: S \rightarrow \varepsilon^{-1}(\ulcorner) \subseteq T$ is unique; the map $f$ is therefore determined by $\varepsilon$.

In an inclusion $(f, \varepsilon): S \rightarrow T$, the events in $f(S)=\varepsilon^{-1}(\ulcorner)$ are executing, whereas the events in $T \backslash f(S)$ are either not started or terminated. In the composition $(g \circ f, \eta), \eta$ is defined such that events in $U \backslash g(T)$ retain their status from the inclusion of $T$ in $U$, events properly in $T$ preserve their status from the inclusion of $S$ in $T$, and events coming from $S$ are executing. See Figure 9 for an example.

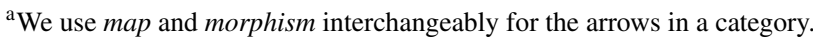


Figure 9. Composition of morphisms $S \rightarrow T \rightarrow U$ in $\square$

$T \quad U$

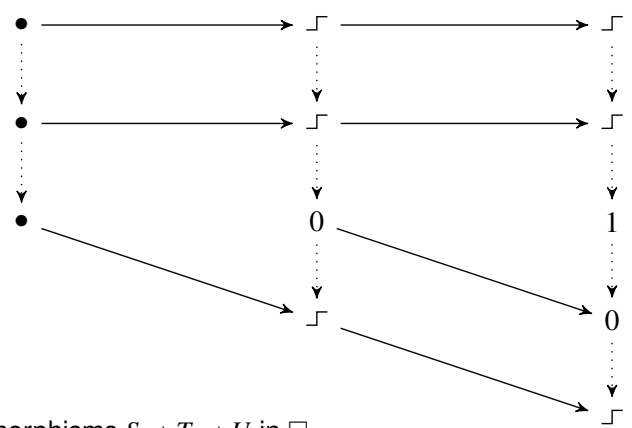

For each $n \geq 1$ denote by $[n]$ the linear poset

$$
[n]=\{1-\rightarrow 2-\rightarrow \cdots-\rightarrow n\},
$$

together with $[0]=\emptyset$.

Definition 2. The precube category is the full subcategory $\square \subseteq \square$ on objects $[n]$ for all $n \geq 0$.

Proposition 3. The category $\square$ is skeletal, and the inclusion $\square \subseteq \square$ is an equivalence of categories and admits a unique left inverse.

Proof. It is clear that $\square$ contains no non-trivial isomorphisms, hence is skeletal. For every $S \in \square$ of cardinality $|S|=n$ there is a unique isomorphism $\imath_{S}: S \rightarrow[n]$ in $\square$. Hence there is a unique functor $\rho: \square \rightarrow \square$, which is a left inverse of the inclusion $\square \subseteq \square$; it is given by $\rho(S)=[|S|]=[n]$ on objects and for any $(f, \varepsilon): S \rightarrow T$ by $\rho(f, \varepsilon)=\left(\iota_{T} \circ f \circ l_{S}^{-1}, \varepsilon \circ l_{T}^{-1}\right)$ on morphisms.

Remark 4. The construction above mimics the situation for the base category of presimplicial sets. Let $\Delta$ be the full subcategory of Pos spanned by the linear posets and $\Delta \subseteq \Delta$ the full subcategory on objects $[n]$ for $n \geq 0$. Except for the maps being injective, $\Delta$ is the augmented simplex category, see [28, VII.5], and presheaves on $\Delta$, i.e., functors from the opposite category $\Delta^{\mathrm{op}}$ into Set, are presimplicial sets. The category $\Delta$ is skeletal, and the inclusion $\Delta \subseteq \Delta$ is an equivalence of categories and admits a unique left inverse. Consequently, the presheaf categories $\operatorname{Set}^{\Delta^{o p}}$ and $\mathrm{Set}^{\mathrm{A}}{ }^{\mathrm{oP}}$ are uniquely naturally isomorphic, and one may be used as drop-in replacement of the other. See [31] for more discussion on this subject.

Remark 5. A similar base category is introduced in [3] for cubical homotopy type theory, see also $[1,4]$. Let $\mathscr{B}$ be the category with objects linear posets and morphisms in $\mathscr{B}(S, T)$ those functions $f: S \rightarrow T \sqcup\{0,1\}$ (disjoint union) for which the restrictions $f_{\mid f^{-1}(T)}$ to elements which do not map to 0 or 1 are poset isomorphisms. Then $\mathscr{B}=\square^{\text {op }}$, as any $f \in \mathscr{B}(S, T)$ is uniquely determined by $\varepsilon: S \rightarrow\{0,\ulcorner, 1\}$ given by

$$
\varepsilon(x)= \begin{cases}\ulcorner & \text { if } f(x) \in T, \\ f(x) & \text { if } f(x) \in\{0,1\} .\end{cases}
$$

The category defined in [3] uses unordered sets and also permits morphisms $f$ for which $f_{\mid f^{-1}(T)}$ is merely injective. These two extensions are independent of each other; removing the order amounts 
to introducing symmetries, and removing surjectivity equips precubical sets with degeneracies (thus passing to cubical sets). See [20] for the presheaf categories of cubical and symmetric cubical sets.

We proceed to show that the (reduced) precube category $\square$ is isomorphic to the standard base category for precubical sets $[19,20]$. For any $n \geq 1, i \in[n]$, and $v \in\{0,1\}$, define a $\square$-map $d_{i, n}^{v}=$ $\left(d_{i, n}, \varepsilon_{i}^{V}\right):[n-1] \rightarrow[n]$ by

$$
d_{i, n}(k)=\left\{\begin{array}{ll}
k & \text { for } 1 \leq k<i, \\
k+1 & \text { for } i \leq k \leq n-1
\end{array} \quad \text { and } \quad \varepsilon_{i}^{v}(k)= \begin{cases}v & \text { for } k=i, \\
\ulcorner & \text { for } k \neq i .\end{cases}\right.
$$

Lemma 6. Let $(f, \varepsilon) \in \square([m],[n]), m<n$, and let $s=\max \{i \in[n] \mid i \notin f([m])\}$. Then $(f, \varepsilon)=$ $d_{s, n}^{\varepsilon(s)} \circ(g, \zeta)$, where $(g, \zeta) \in \square([m],[n-1])$ is given by

$$
g(i)=\left\{\begin{array}{ll}
f(i) & \text { for } f(i)<s, \\
f(i-1) & \text { for } f(i)>s
\end{array} \quad \text { and } \quad \zeta(j)= \begin{cases}\varepsilon(j) & \text { for } j<s, \\
\varepsilon(j+1) & \text { for } j \geq s .\end{cases}\right.
$$

Proof. Elementary calculations.

Lemma 7. Let $(f, \varepsilon) \in \square([n-s],[n])$ and denote $[n] \backslash f([n-s])=\left\{a_{1}<\cdots<a_{s}\right\}$. Then

$$
(f, \varepsilon)=d_{a_{s}, n}^{\varepsilon\left(a_{s}\right)} \circ d_{a_{s-1}, n-1}^{\varepsilon\left(a_{s-1}\right)} \circ \cdots \circ d_{a_{2}, n-s+2}^{\varepsilon\left(a_{2}\right)} \circ d_{a_{1}, n-s+1}^{\varepsilon\left(a_{1}\right)} .
$$

Proof. From Lemma 6 by induction.

Proposition 8. The category $\square$ is generated by morphisms $d_{i, n}^{v}$ and the co-precubical identities $d_{j, n}^{v} \circ d_{i, n-1}^{\mu}=d_{i, n}^{\mu} \circ d_{j-1, n-1}^{v}$ for $1 \leq i<j<n$ and $v, \mu \in\{0,1\}$. Every $\square$-map $[n-s] \rightarrow[n]$ can be written uniquely as a composition

$$
d_{a_{1}, \ldots, a_{s}}^{v_{1}, \ldots, v_{s}}=d_{a_{s}, n}^{v_{s}} \circ d_{a_{s-1}, n-1}^{v_{s-1}} \circ \cdots \circ d_{a_{2}, n-s+2}^{v_{2}} \circ d_{a_{1}, n-s+1}^{v_{1}}
$$

where $v_{i} \in\{0,1\}$ and $1 \leq a_{1}<\cdots<a_{s} \leq n$.

Proof. Lemma 7 implies that every morphism $(f, \varepsilon) \in \square([m],[n])$ can be presented as a composition of elementary morphisms in which the sequence of lower indices is strictly decreasing. Such presentations are unique since $f$ can be recovered from the sequences $a_{s}, \ldots, a_{1}$ and $\varepsilon\left(a_{s}\right), \ldots, \varepsilon\left(a_{1}\right)$. It remains to show that the co-precubical relations hold, which is elementary.

\subsection{Precubical Sets}

Precubical sets are usually defined as presheaves over $\square$, i.e., functors $\square^{\mathrm{op}} \rightarrow$ Set [19]. Using Proposition 3, we may instead use presheaves over $\odot$ :

Proposition 9. The presheaf categories $\mathrm{Set}^{\square \mathrm{op}}$ and $\mathrm{Set}^{\square \mathrm{op}}$ are uniquely naturally isomorphic.

Proof. Each functor $F: \square^{\mathrm{op}} \rightarrow$ Set extends uniquely to a functor $\dot{F}=\rho \circ F: \square^{\mathrm{op}} \rightarrow$ Set by composition with the functor $\rho$ from the proof of Proposition 3. The functor $\dot{F}$, in turn, restricts to $F$ on $\square$ op. 
Definition 10. The category of precubical sets is the presheaf category Set ${ }^{\mathrm{op}}$ or, equivalently, $\mathrm{Set}^{\oplus^{\mathrm{op}}}$. That is, a precubical set is a functor $\square^{\mathrm{op}} \rightarrow$ Set or $\square^{\mathrm{op}} \rightarrow$ Set, and a precubical map is a natural transformation of precubical sets.

We write $X_{n}$ for $X([n]), \delta_{\left\{a_{1}, \ldots, a_{s}\right\}}^{v_{1}, \ldots, v_{s}}$ for $X\left(d_{a_{1}, \ldots, a_{s}}^{v_{1}, \ldots, v_{s}}\right)$, and $\delta_{\left\{a_{1}, \ldots, a_{s}\right\}}^{v}$ for $X\left(d_{a_{1}, \ldots, a_{s}}^{v, \ldots, v}\right)$. The map $X\left(d_{i, n}^{v}\right): X_{n} \rightarrow X_{n-1}$ is denoted by $\delta_{i}^{v}$. For any $x \in X_{n}, n$ is called the dimension of $x$ and indicated by $\operatorname{dim} x=n$. The maps $\delta_{i}^{v}$ are called elementary face maps and the maps $\delta_{\left\{a_{1}, \ldots, a_{s}\right\}}^{v_{1}, \ldots, v_{s}}$, face maps.

Definition 11. The standard $S$-cube on a linear poset $(S,-\rightarrow)$ is the precubical set $\square^{S}$, where

- $\square$ is the set of functions $x: S \rightarrow\{0,\ulcorner, 1\}$ taking value $\ulcorner$ on exactly $k$ elements;

- $\delta_{i}^{v}$ converts the $i$-th occurence of $\left\ulcorner\right.$ into $v \in\{0,1\}$, i.e., if $x^{-1}\left(\ulcorner)=\left\{p_{1}-\rightarrow \cdots-\rightarrow p_{k}\right\}\right.$, then

$$
\delta_{i}^{v} x(p)= \begin{cases}v & \text { for } p=p_{i} \\ x(p) & \text { otherwise }\end{cases}
$$

Every function $x: S \rightarrow\left\{0,\ulcorner, 1\}\right.$ in $\square_{k}^{S}$ determines a unique poset map $f_{x}:[k] \rightarrow S$ by the isomorphism $f_{x}:[k] \rightarrow f_{x}([k])=x^{-1}\left(\ulcorner)\right.$. Denote the unique top-dimensional cell of $\square^{S}$ (the unique element of $\left.\square^{S}(S)\right)$ by $\mathbf{y}_{S}$. Then $\mathbf{y}_{S}(x)=\ulcorner$ for all $x \in S$. The order on $S$ is necessary to define face maps: it determines which of the $\ulcorner$ values should be converted into $v$.

For $n \geq 0$, the standard $n$-cube is $\square^{n}:=\square^{[n]}$, and its unique $n$-cell is denoted by $\mathbf{y}_{n}$.

Regarded as a presheaf, $\square^{S}$ is the functor represented by $S$, i.e., $\square^{S}(T)=\square(T, S)$. The cell $\mathbf{y}_{S}$ corresponds to the identity morphism on $S$. The following is an immediate consequence of the Yoneda lemma.

Lemma 12. Let $X$ be a precubical set and $x \in X_{n}$. Then there exists a unique precubical map $\mathbf{i}_{x}: \square^{n} \rightarrow X$ such that $\mathbf{i}_{x}\left(\mathbf{y}_{n}\right)=x$.

\subsection{Labelings and Events}

Definition 13. Let $A$ be a finite set. The labeling object on $A$ is the precubical set $! A$ with $! A_{n}=A^{n}$ and $\delta_{i}^{v}$ defined by

$$
\delta_{i}^{v}\left(\left(a_{1}, \ldots, a_{n}\right)\right)=\left(a_{1}, \ldots, a_{i-1}, a_{i+1}, \ldots, a_{n}\right) .
$$

The event object on $A$ is the precubical subset $! ! A \subseteq !$ g given by

$$
! ! A_{n}=\left\{\left(a_{1}, \ldots, a_{n}\right) \mid a_{i} \neq a_{j} \text { whenever } i \neq j\right\} .
$$

Regarded as a presheaf, $! A(S)=\operatorname{Set}(S, A)$, hence $! A$ is representable in Set via the forgetful functor $\square \rightarrow$ Set. In particular, $! A_{n}$ is exactly the set of isomorphism classes of linear posets over $A$ with $n$ elements. Similarly, !!A(S)= $\operatorname{Inj}(S, A)$, where Inj is the category of sets and injective maps, so that !!A is representable in Inj via the forgetful functor $\square \rightarrow \operatorname{Inj}$. Also note that $! A$ is infinite, whereas !!A is finite: if $A$ has $m$ elements, then $! ! A_{m}$ consists of all permutations of these elements and $! ! A_{n}=\emptyset$ for $n>m$.

Every function $f \in \operatorname{Set}(A, B)$ induces a precubical map $! f: ! A \rightarrow ! B$, and every injective function $g \in \operatorname{Inj}(A, B)$ induces a precubical map $! ! g: ! ! A \rightarrow ! ! B$, turning them into functors ! : Set $\rightarrow$ $\operatorname{Set}^{\square \text { op }}$ and !!:Inj $\rightarrow \operatorname{Inj}^{\square^{\text {op }}}$. These are left adjoint to the functors Set ${ }^{\square^{\text {op }}} \rightarrow$ Set and Inj ${ }^{\square^{\text {op }}} \rightarrow \operatorname{Inj}$ mapping $X$ to $X_{1}$, hence !A and !!A are free in the following sense. 


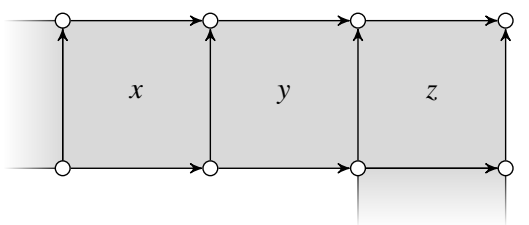

Figure 10. Example of a precubical set that is not event consistent (left and bottom right edges identified).

Lemma 14. Let $X$ be a precubical set and $A$ a finite set.

(1) Any function $\lambda_{1}: X_{1} \rightarrow A$ for which $\lambda_{1}\left(\delta_{1}^{0} x\right)=\lambda_{1}\left(\delta_{1}^{1} x\right)$ and $\lambda_{1}\left(\delta_{2}^{0} x\right)=\lambda_{1}\left(\delta_{2}^{1} x\right)$ for all $x \in$ $X_{2}$ extends uniquely to a precubical map $\lambda: X \rightarrow ! A$.

(2) Any function $\mathrm{ev}_{1}: X_{1} \rightarrow A$ for which $\operatorname{ev}_{1}\left(\delta_{1}^{0} x\right)=\operatorname{ev}_{1}\left(\delta_{1}^{1} x\right), \operatorname{ev}_{1}\left(\delta_{2}^{0} x\right)=\operatorname{ev}_{1}\left(\delta_{2}^{1} x\right)$, and $\mathrm{ev}_{1}\left(\delta_{1}^{0} x\right) \neq \mathrm{ev}_{1}\left(\delta_{2}^{0} x\right)$ for all $x \in X_{2}$ extends uniquely to a precubical map ev $: X \rightarrow ! ! A$.

Proof. For the first claim, define functions $f_{i}:[1] \rightarrow[n]$, for all $n \geq 0$ and $i \in[n]$, by $f_{i}(1)=i$. Then define $\lambda$ by $\lambda(x)=\left(\lambda_{1}\left(\left(f_{1}, \varepsilon_{1}\right)^{\mathrm{op}}(x)\right), \ldots, \lambda_{1}\left(\left(f_{n}, \varepsilon_{n}\right)^{\mathrm{op}}(x)\right)\right)$ for $x \in X_{n}$. Because of $\lambda_{1} \circ \delta_{i}^{0}=$ $\lambda_{1} \circ \delta_{i}^{1}$, the choices of $\varepsilon_{i}$ do not matter. It is clear that $\lambda$ is the unique extension of $\lambda_{1}$.

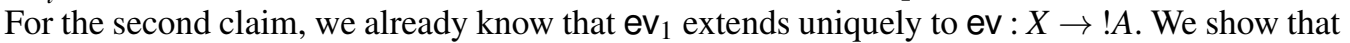
the image of ev lies in !!A. With a slight abuse of notation, write $\mathrm{ev}(x)=\left(\operatorname{ev}_{1}(x), \ldots, \mathrm{ev}_{n}(x)\right)$ for $x \in X_{n}$, and suppose there exists an $x \in X_{n}$ with $\operatorname{ev}_{i}(x)=\operatorname{ev}_{j}(x)$ for $i<j$. Let $(f, \varepsilon) \in \square([2],[n])$ be the morphism that satisfies $f(1)=i$ and $f(2)=j$ ( $\varepsilon$ is again irrelevant), and let $y=(f, \varepsilon)^{\mathrm{op}}(x) \in$ $X_{2}$. Then $\mathrm{ev}_{1}\left(\delta_{1}^{0} y\right)=\mathrm{ev}_{i}(x)=\mathrm{ev}_{j}(x)=\mathrm{ev}_{1}\left(\delta_{2}^{0} y\right)$, in contradiction to the second property of $\mathrm{ev}_{1}$.

Let henceforth $\Sigma$ be a fixed finite set.

Definition 15. Let $X$ be a precubical set. A labeling of $X$ is a precubical map $\lambda: X \rightarrow ! \Sigma$. An event identification on $X$ is a map ev $: X \rightarrow ! ! \Sigma$.

Every event identification on $X$ is also a labeling on $X$, but the converse does not hold; in fact, all precubical sets admit labelings, but not necessarily event identifications:

Example 16. Figure 10 shows a precubical set with three 2-cells $x, y, z$ and $\delta_{1}^{0} x=\delta_{2}^{0} z$. Any event identification ev $: X \rightarrow ! ! \Sigma$ must fulfill

$$
\mathrm{ev}\left(\delta_{2}^{0} z\right)=\operatorname{ev}\left(\delta_{1}^{0} x\right)=\operatorname{ev}\left(\delta_{1}^{1} x\right)=\operatorname{ev}\left(\delta_{1}^{0} y\right)=\operatorname{ev}\left(\delta_{1}^{1} y\right)=\operatorname{ev}\left(\delta_{1}^{0} z\right)
$$

a contradiction.

Definition 17. A precubical set $X$ is event consistent if it admits an event identification ev :X $\rightarrow$ $! ! \Sigma$.

Lemma 18. A precubical set $X$ is event consistent iff there exists an equivalence relation $\sim$ on $X_{1}$ such that for all $x \in X_{2}, \delta_{1}^{0} x \sim \delta_{1}^{1} x, \delta_{2}^{0} x \sim \delta_{2}^{1} x$, and $\delta_{1}^{0} x \not \delta_{2}^{0} x$.

Proof. First suppose that $X$ is event consistent. Let ev $: X \rightarrow ! ! \Sigma$ and define the equivalence relation $\sim$ on $X_{1}$ by $x \sim y$ iff $\operatorname{ev}(x)=\operatorname{ev}(y)$. From the definition of ! $\Sigma$ we have $\operatorname{ev}\left(\delta_{i}^{0} x\right)=\delta_{i}^{0} \operatorname{ev}(x)=$ $\delta_{i}^{1} \operatorname{ev}(x)=\operatorname{ev}\left(\delta_{i}^{1} x\right)$ and therefore $\delta_{i}^{0} x \sim \delta_{i}^{1} x$ holds for all $x \in X_{2}$ and for $i \in\{1,2\}$. From the definition of !! $\Sigma$ we have $\operatorname{ev}\left(\delta_{1}^{0} x\right)=\delta_{1}^{0} \operatorname{ev}(x) \neq \delta_{2}^{0} \operatorname{ev}(x)=\operatorname{ev}\left(\delta_{2}^{0} x\right)$ and therefore $\delta_{1}^{0} x \neq \delta_{2}^{0} x$ holds for all $x \in X_{2}$. 
For the other direction, suppose there is a relation $\sim$ that satisfies the properties in the lemma and let $\Sigma=X_{1} / \sim$ be the set of equivalence classes. The quotient map $X_{1} \rightarrow \Sigma$ then extends uniquely to ev : $X \rightarrow ! ! \Sigma$ by Lemma 14 , which yields the event identification needed.

Any event consistent precubical set admits a smallest equivalence relation owing to Lemma 18 , denoted $\sim_{\mathrm{ev}}$. It is given as the transitive closure of $\left\{\left(\delta_{i}^{0} x, \delta_{i}^{1} x\right) \mid x \in X_{2}, i \in\{1,2\}\right\}$, we call its equivalence classes the universal events of $X$.

We will generally only concern ourselves with event consistent precubical sets in the rest of this work, but come back to the more general case at the end of Section 7.1.

Example 19. The standard $S$-cube $\square^{S}$ from Definition 11 is event consistent for any linear poset $S$. Its universal event equivalence is given by $x \sim_{\mathrm{ev}} y$ iff $x^{-1}\left(\ulcorner)=y^{-1}(\ulcorner)\right.$, induced by the event identification ev : $\square^{S} \rightarrow ! ! S$ with $\operatorname{ev}(x)=x^{-1}\left(\ulcorner)\right.$. If $X$ is a precubical subset of $\square^{S}$, then $X$ is also event consistent. Such precubical subsets of standard cubes are called sculptures in [9], where it is shown that they correspond to Chu spaces over $\{0,\ulcorner, 1\}[35,36]$.

The term "universal events" is justified by the following factorization property, which follows immediately from the definitions.

Proposition 20. Every labeling $\lambda: X \rightarrow ! \Sigma$ factors uniquely through $E_{X}$, i.e., there is a unique factorization $\lambda_{1}=\lambda_{1}^{\mathrm{ev}} \circ \mathrm{ev}_{1}: X_{1} \rightarrow E_{X} \rightarrow \Sigma$ that extends to a factorization $\lambda=\lambda^{\mathrm{ev}} \circ \mathrm{ev}: X \rightarrow$ $! ! E_{X} \rightarrow ! \Sigma$.

Hence also any event identification factors uniquely through the universal events.

Proposition 21. Let $\lambda: X \rightarrow ! \Sigma$ be a labeling. If $x \in X_{n}$ and $(f, \varepsilon) \in \square([m],[n])$, then $\lambda\left((f, \varepsilon)^{\mathrm{op}}(x)\right)=\lambda(x) \circ f$. In particular,

$$
\lambda\left(\delta_{i}^{v} x\right)=\left(\lambda_{1}(x), \ldots, \lambda_{i-1}(x), \lambda_{i+1}(x), \ldots, \lambda_{n}(x)\right) .
$$

Proof. Straightforward from the definitions.

Lemma 22. Let $X$ be an event consistent precubical set, $n \geq 0, x \in X_{n}, A, B \subseteq[n]$, and $v \in\{0,1\}$. Then $\delta_{A}^{v} x=\delta_{B}^{v} x$ implies $A=B$.

Proof. Applying Proposition 21 to ev $: X \rightarrow ! ! E_{X}$ yields ev $\left(\delta_{A}^{v} x\right)=\left(\mathrm{ev}_{i}(x)\right)_{i \in[n] \backslash A}$ and $\mathrm{ev}\left(\delta_{B}^{v} x\right)=$ $\left(\mathrm{ev}_{i}(x)\right)_{i \in[n] \backslash B}$. Since events $\mathrm{ev}_{i}(x)$ are pairwise distinct, we obtain $A=B$.

Remark 23. For $n=2$, the above lemma reduces to the definition of event consistency. The lemma will show its importance once we consider tracks, i.e., sequences of cells connected at faces, in Section 5. There is a related property of being non-selfintersecting which has been used for the same purpose, see for example [11]: a precubical set $X$ is non-selfintersecting if $\delta_{A}^{v} x=\delta_{B}^{\mu} x$ implies $A=B$ and $v=\mu$ for all $x \in X$. Example 16 shows that precubical sets may be non-selfintersecting, but not event consistent; similarly, event consistency does not imply the non-selfintersecting property, see [9]. Finally, also Section 4 of [41] contains some precursors to our notion of event consistency.

If $f: X \rightarrow Y$ is a precubical map, then $x \sim_{\mathrm{ev}} y$ implies $f(x) \sim_{\mathrm{ev}} f(y)$ for $x, y \in X_{1}$, and $f_{1}: X_{1} \rightarrow$ $Y_{1}$ induces a map $E_{f}: E_{X} \rightarrow E_{Y}$. This defines a functor $E:$ Set $^{\square \text { op }} \rightarrow$ Set. 


\subsection{Higher-Dimensional Automata}

Higher-dimensional automata are labeled precubical sets with initial and accepting cells.

Definition 24. $A$ labeled precubical set is an event consistent precubical set $X$ together with a labeling $\lambda: X \rightarrow ! \Sigma$. Maps $f: X \rightarrow Y$ of labeled precubical sets preserve labelings: $\lambda_{X}=\lambda_{Y} \circ f$.

That is to say, the category of labeled precubical sets is the full subcategory of the slice category Set $^{\square^{\mathrm{op}}} / ! \Sigma$ on event consistent objects.

Definition 25. Let $(X, \lambda)$ be a labeled precubical set. The label of a cell $x \in X_{n}$ is the linear pomset $\ell(x)=\left(\mathrm{ev}(x),-\rightarrow, \lambda_{\mathrm{ev}(x)}\right)$ with

$$
\mathrm{ev}(x)=\left(\mathrm{ev}_{1}(x) \rightarrow \mathrm{ev}_{2}(x) \rightarrow-\rightarrow \cdots \rightarrow \mathrm{ev}_{n}(x)\right)
$$

and $\lambda_{\mathrm{ev}(x)}\left(\mathrm{ev}_{i}(x)\right)=\lambda_{i}(x)$

Note that $\ell(x)$ is basically the tuple $\lambda(x)$, but using Proposition 20 we now regard it as a pomset. Event consistency is essential for this to make sense. Of the two following elementary lemmas, the first follows from Proposition 21 and Lemma 18; the second one is trivial.

Lemma 26. If $x$ is a face of $y$, then there is a pomset inclusion $\ell(x) \subseteq \ell(y)$.

Lemma 27. Let $f: X \rightarrow Y$ be a map of labeled precubical sets and $x \in X_{n}$. Then $\ell(f(x))=$ $E_{f}(\ell(x)) \cong \ell(x)$.

Definition 28. A higher-dimensional automaton (HDA) is a tuple $(X, I, F, \lambda)$, where $(X, \lambda)$ is a labeled precubical set, $I \subseteq X$ is a set of initial cells and $F \subseteq X$ a set of accepting cells. If $X$ and $Y$ are HDAs, then a precubical map $f: X \rightarrow Y$ is an HDA map if it preserves labels and initial and accepting cells: $\lambda_{X}=\lambda_{Y} \circ f, f\left(I_{X}\right) \subseteq I_{Y}$, and $f\left(F_{X}\right) \subseteq F_{Y}$.

\section{Pomsets with Interfaces}

We now return to posets and pomsets and introduce interfaces for them, building on our work in [8] but enriched with event orders. Recall that $\Sigma$ is a fixed finite set.

\subsection{Ipomsets}

Definition 29. An ipomset is a tuple $\left(P,<_{P},-\rightarrow_{P}, \lambda_{P}, S_{P}, T_{P}\right)$, where $P$ is a finite set;

- $<_{P}$ is a strict partial order on $P$ called precendence order;

$-\rightarrow P$ is a strict partial order on $P$ called event order;

- $\lambda_{P}: P \rightarrow \Sigma$ is a function called labeling;

- $S_{P}$ is a subset of the <-minimal elements of $P$ called source set;

- $T_{P}$ is a subset of the <-maximal elements of $P$ called target set.

We require that the relation $<_{P} \cup--\rightarrow P_{P}$ is total: if $x \neq y \in P$, then $x$ and $y$ are comparable by $<_{P}$ or by $\rightarrow \rightarrow P$.

Note that $<_{P} \cup \rightarrow \rightarrow_{P}$ need not be a partial order, see Example 32 below. The linear pomset $\left(S_{P},-\rightarrow P \cap\left(S_{P} \times S_{P}\right), \lambda_{P \mid S_{P}}\right)$, where $\lambda_{P \mid S_{P}}$ denotes the domain restriction of $\lambda_{P}: P \rightarrow \Sigma$ to $S_{P}$, is called source interface of $P$; we often simply write $\left(S_{P},-\rightarrow_{P}, \lambda_{P}\right)$. Similarly, $\left(T_{P},-\rightarrow_{P}, \lambda_{P}\right)$ is the 
target interface of $P$. If $S=T=\emptyset$, then $P$ is a pomset in the classical sense [17, 33] (ignoring the event order). If $S=T=\emptyset$ and $<$ is linear, then $P$ corresponds to a string.

Remark 30. In [8] we defined ipomsets without an event order. Instead we picked out sources and targets using injections $s:[n] \rightarrow P$ and $t:[m] \rightarrow P$. This implicitly defines (linear) event orders on the subsets $S_{P}=s([n]) \subseteq P$ and $T_{P}=t([m]) \subseteq P$, so the only essential difference between [8] and our present setting is that the event order is extended to the whole of $P$. When $S_{P} \cap T_{P}=\emptyset$, such an extension is always possible; and we will see later that the ordered structures properly corresponding to HDAs are our present event-ordered ipomsets, see Definitions 55 and 60.

Remark 31. The ordered structure $\left(P,<_{P},-\rightarrow_{P}\right)$ underlying an ipomset is a biposet in the sense of [5], but because of the requirement that $<_{P} \cup-\rightarrow_{P}$ be total, not all biposets may be used. [5] is concerned with $n$-posets, i.e., finite sets with $n$ partial orders, and then introduces a notion of higher-dimensional automata as recognizers of such structures. Except for the name, the higherdimensional automata of [5] have nothing to do with our HDAs.

Example 32. By definition, the maximal antichains of the precedence order are linearly ordered by the event order, but the event order may contain further arrows. As an example, consider the ipomset

$$
P=\left(\begin{array}{l}
a \longrightarrow b \\
\hat{c}^{-}
\end{array}\right)
$$

with precedence order $a<b$ and event order $b \rightarrow-\rightarrow-\rightarrow a$. Both maximal antichains $a \| c$ and $b \| c$ are linearly $-\rightarrow-$-ordered, but by transitivity, also $b \rightarrow a$.

Let $Q \subseteq P$ be a subset of the ipomset $(P,<,-\rightarrow, \lambda, S, T)$. Then the restriction

$$
P_{\mid Q}:=\left(Q,<\cap(Q \times Q),-\rightarrow \cap(Q \times Q), \lambda_{\mid Q}, S \cap Q, T \cap Q\right)
$$

is also an ipomset.

Definition 33. Ipomsets $P$ and $Q$ are isomorphic if there exists a bijection $f: P \rightarrow Q$ (an ipomset isomorphism) that

- respects precedence: for all $x, y \in P, x<_{P}$ y iff $f(x)<_{Q} f(y)$;

- respects essential event ordering: for all $x, y \in P$ with $x \|_{P} y, x \rightarrow \rightarrow_{P}$ y iff $f(x) \rightarrow_{Q} f(y)$;

- respects labels and interfaces: $\lambda_{Q} \circ f=\lambda_{P}, f\left(S_{P}\right)=S_{Q}$, and $f\left(T_{P}\right)=T_{Q}$.

By definition, $f$ is only required to respect the part of the event ordering which orders events in antichains. In Section 4.4 we will introduce a notion of morphism between ipomsets for which the above form the isomorphisms.

Isomorphisms between ipomsets are unique:

Lemma 34. There is at most one isomorphism between any two ipomsets.

Proof. Using poset filtrations, we can combine the two orders on an ipomset into a linear order and then use the fact that isomorphisms between linearly ordered sets are unique:

Let $P$ be an ipomset and $P_{0}$ its set of $<_{P}$-minimal elements. Let $P_{1}$ be the set of $<_{P}$-minimal elements of the sub-ipomset $P \backslash P_{0}, P_{2}$ the set of $<_{p}$-minimal elements of $P \backslash P_{0} \backslash P_{1}$, and so on. The finite disjoint union $P=P_{0} \sqcup P_{1} \sqcup P_{2} \sqcup \ldots$ is called filtration of $P$. (More precisely, one can 
set $P_{>-1}=P$ and then inductively for $i \geq 0$, until exhaustion, let $P_{i}$ be the $<_{P}$-minimal elements of $P_{>i-1}$ and $P_{>i}=P_{>i-1} \backslash P_{i}$.)

Now all $P_{i}$ are $<_{P}$-antichains and hence linearly ordered by $\rightarrow \rightarrow_{P}$. Let $\prec_{P}$ be the relation on $P$ defined by $x \prec_{P} y$ if $x \in P_{i}$ and $y \in P_{j}$ for $i<j$, or $x, y \in P_{i}$ for some common $i$ and $x \rightarrow P y$. Then $\prec_{P}$ is a linear order on $P$. Further, if $f: P \rightarrow Q$ is an ipomset isomorphism, then $f:\left(P, \prec_{P}\right) \rightarrow$ $(Q, \prec Q)$ is an isomorphism of linear orders; hence $f$ is unique.

\subsection{Gluing and Parallel Compositions}

The gluing composition $P * Q$ of two ipomsets is defined if the target interface of $P$ is isomorphic to the source interface of $Q$, in which case it identifies the targets of $P$ with their corresponding sources in $Q$ and makes all non-interface elements in $P$ precede all non-interface elements in $Q$. Below, $(\cdot)^{+}$is used for transitive closure.

Definition 35. Let $P$ and $Q$ be ipomsets such that $\left(T_{P},-\rightarrow P, \lambda_{P}\right)$ is isomorphic to $\left(S_{Q},-\rightarrow{ }_{Q}, \lambda_{Q}\right)$. The gluing composition of $P$ and $Q$ is $P * Q=\left(P \sqcup\left(Q \backslash S_{Q}\right),<,-\rightarrow, \lambda, S_{P}, T_{Q}\right)$ with $<,-\rightarrow$, and $\lambda$ defined as follows:

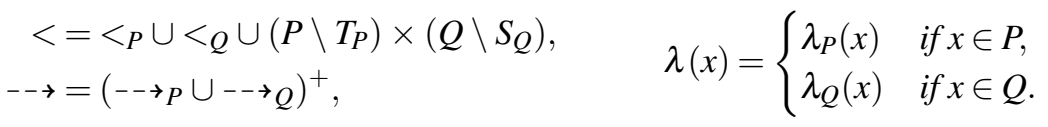

It is clear that $P * Q$, if defined, is indeed again an ipomset: the only non-trivial property to check is irreflexivity of $\rightarrow \rightarrow$, which follows from the fact that the restrictions of $\rightarrow$ to $P$ and $Q$ are precisely $\rightarrow \rightarrow_{P}$ and $\rightarrow Q$, respectively. It is also clear that gluing composition respects isomorphisms:

Lemma 36. If $P \cong P^{\prime}$ and $Q \cong Q^{\prime}$, then $P * Q$ is defined iff $P^{\prime} * Q^{\prime}$ is, and in that case, $P * Q \cong$ $P^{\prime} * Q^{\prime}$.

Gluing composition of pomsets, i.e., ipomsets with empty interfaces $S_{P}=T_{P}=\emptyset$, is the same as the standard serial composition $[17,18]$. Gluing composition of strings is concatenation. We also introduce a parallel composition of ipomsets that generalizes the eponymous operation for pomsets.

Definition 37. The parallel composition of ipomsets $P$ and $Q$ is $P \| Q=(P \sqcup Q,<,-\rightarrow, \lambda$, $\left.S_{P} \sqcup S_{Q}, T_{P} \sqcup T_{Q}\right)$ with $<,-\rightarrow$, and $\lambda$ defined as follows:

$$
\begin{aligned}
< & =<_{P} \cup<_{Q}, \\
-\rightarrow & =-\rightarrow_{P} \cup-\rightarrow_{Q} \cup P \times Q,
\end{aligned} \quad \lambda(x)= \begin{cases}\lambda_{P}(x) & \text { if } x \in P, \\
\lambda_{Q}(x) & \text { if } x \in Q .\end{cases}
$$

It is easy to see that $P \| Q$ is again an ipomset. Parallel composition of ipomsets is not commutative because of the event order. It is again clear that parallel composition respects isomorphisms:

Lemma 38. If $P \cong P^{\prime}$ and $Q \cong Q^{\prime}$, then $P\left\|Q \cong P^{\prime}\right\| Q^{\prime}$.

\subsection{Interval Orders}

An interval order is a poset $P$ in which $x<z$ and $y<w$ imply $x<w$ or $y<z$ for all $x, y, z, w \in P$. 


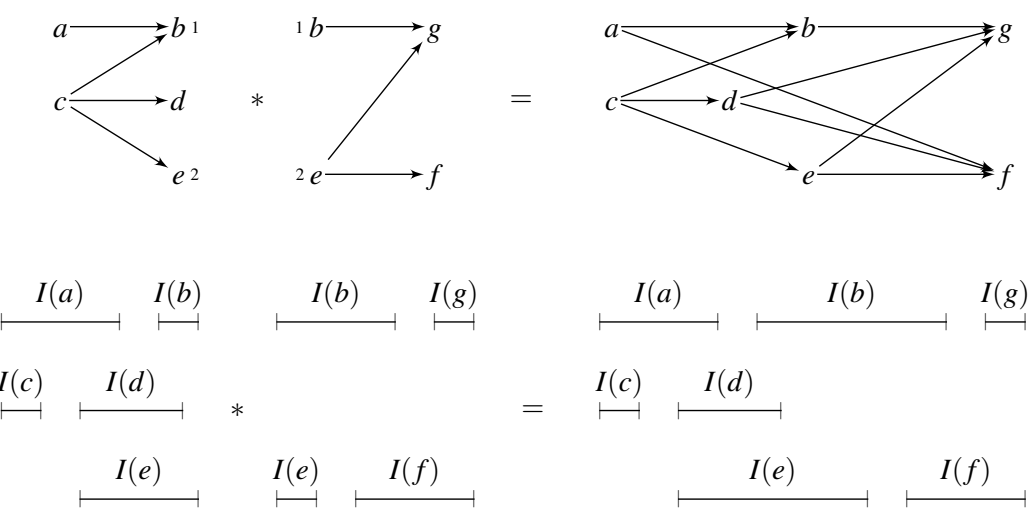

Figure 11. Two interval ipomsets and their gluing: above as ipomsets, below using interval representations (event order not shown)

Lemma 39 ([15, 16, 22]). The following are equivalent for any poset $P$ :

(1) $P$ is an interval order;

(2) $P$ does not contain an induced subposet $2+2=(\bullet \longrightarrow \bullet)$;

(3) $P$ has an interval representation: a pair of functions $s, t: P \rightarrow Q$ into a linear poset $\left(Q,<_{Q}\right)$ such that for all $x, y \in P, s(x)<_{Q} t(x)$, and $x<_{P} y$ iff $t(x)<_{Q} s(y)$;

(4) the order $\prec$ on maximal antichains of $P$ defined by $X \prec Y$ if $X \neq Y$ and $y \nless_{P} x$ for all $x \in X$, $y \in Y$ is linear.

Definition 40. An interval ipomset is an ipomset $P$ for which the underlying precedence poset $\left(P,<_{P}\right)$ is an interval order.

Restrictions of interval ipomsets are again interval. The following is shown in [8] using interval representations, see Figure 11 for an example.

Lemma 41. If $P$ and $Q$ are interval ipomsets and $P * Q$ exists, then $P * Q$ is an interval ipomset.

We develop a decomposition property for interval ipomsets which will be useful later.

Definition 42. An ipomset $P$ is discrete if $<_{P}$ is empty (thus, $-_{-} \rightarrow_{P}$ is a linear order). In addition, $P$ is a starter if $T_{P}=P$, and $P$ is $a$ terminator if $S_{P}=P$. A starter $P$ is elementary if $P \backslash S_{P}$ is a singleton, and a terminator $P$ is elementary if $P \backslash T_{P}$ is a singleton.

Starters may be used to start events and terminators to terminate them. In compositions, they can switch off parts of starting or terminating interfaces, see Figure 12.

Every starter is a gluing of elementary starters, and every terminator a gluing of elementary terminators (both not necessarily unique). Identity ipomsets are both starters and terminators, and any discrete ipomset can be written as a gluing of a starter followed by a terminator.

We introduce special notation for discrete ipomsets: for subsets $S, T \subseteq U$ of a linear pomset $(U,-\rightarrow, \lambda)$ we write

$$
{ }_{S} U_{T}=(U, \emptyset,-\rightarrow, \lambda, S, T) .
$$

The next lemma follows easily. 


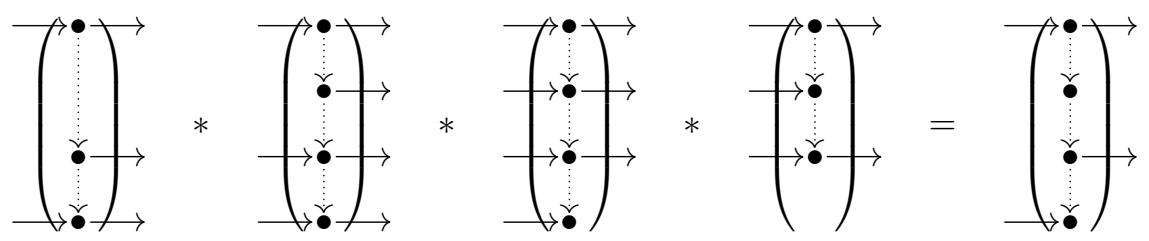

Figure 12. Decomposition of discrete ipomset into elementary starters and terminators.

Lemma 43. Let $S, T$, $U$ be linear pomsets. If $S \subseteq T \subseteq U$, then ${ }_{S} T_{T} *_{T} U_{U} \cong{ }_{S} U_{U}$ and ${ }_{U} U_{T} *_{T} T_{S} \cong$ ${ }_{U} U_{S}$. If $S, T \subseteq U$, then ${ }_{S} U_{U} *{ }_{U} U_{T} \cong{ }_{S} U_{T}$.

Proposition 44. For an ipomset $P$ the following are equivalent:

(1) $P$ is an interval ipomset;

(2) $P$ is a finite gluing of discrete ipomsets;

(3) $P$ is a finite gluing of elementary starters and terminators.

Proof. Equivalence of (2) and (3) is clear. Given that discrete ipomsets are interval, (2) implies (1) by Lemma 41.

To show that (1) implies (2), let $P$ be an interval ipomset and $P_{1} \prec \cdots \prec P_{m}$ the sequence of maximal antichains in $P$ given by Lemma 39(4). Each $P_{i}$ is linearly ordered by the restriction $-\rightarrow_{i}$ of $-\rightarrow P$ to $P_{i}$. Let $S_{1}=S_{P}, T_{m}=T_{P}$, and $T_{i}=S_{i+1}=P_{i} \cap P_{i+1}$ for $i \in[m-1]$, and define ipomsets $P_{i}=\left(P_{i}, \emptyset,-\rightarrow_{i}, \lambda_{\mid P_{i}}, S_{i}, T_{i}\right)$. Then the gluing $Q=P_{1} * \cdots * P_{m}$ is defined; we show that $Q=P$.

It is clear that the underlying sets of $Q$ and $P$ are equal and that the source and target interfaces agree. Further, $-\rightarrow_{Q}=\left(-\rightarrow_{1} \cup \cdots \cup \rightarrow_{m}\right)^{+}=-\rightarrow_{P}$. To see that $<_{Q}=<_{P}$, we note that $x<_{P} y$ implies that $x \in P_{i}$ and $y \in P_{j}$ with $i<j$ and $x, y \notin P_{i} \cap P_{j}$, and vice versa.

Figure 12 shows an example of a decomposition of a discrete ipomset into elementary starters and terminators. This proposition also gives an alternate proof of Lemma 41.

\subsection{Subsumption}

Pomsets may be smoothened, or made less concurrent, by strengthening precedence relations. The corresponding relation between pomsets has been introduced by Grabowski [18] and is nowadays often called subsumption [17]. We adapt it to ipomsets.

Definition 45. An ipomset $Q$ subsumes an ipomset $P$ if there exists a bijection $f: P \rightarrow Q$, called a subsumption map, such that

- for all $x, y \in P, f(x)<_{Q} f(y)$ implies $x<_{P} y$;

- for all $x, y \in P$ with $x \|_{P} y, x \rightarrow P$ y implies $f(x)-\rightarrow Q f(y)$; and

- $\lambda_{Q} \circ f=\lambda_{P}, f\left(S_{P}\right)=S_{Q}$, and $f\left(T_{P}\right)=T_{Q}$.

We write $P \sqsubseteq Q$ if $Q$ subsumes $P$. That is, the points of $P$ and $Q$ are in bijection, but $P$ may be more precedence ordered than $Q$, and $Q$ may be more event ordered than $P$.

If $P$ is discrete or $Q$ is linear, then any subsumption map $f: P \rightarrow Q$ is an isomorphism and, in particular, unique. We extend gluing composition to subsumptions: 
Definition 46. Let $f: P \rightarrow P^{\prime}$ and $g: Q \rightarrow Q^{\prime}$ be subsumption maps and assume $P * Q$ and $P^{\prime} * Q^{\prime}$ to be defined. Define $h=f * g: P * Q \rightarrow P^{\prime} * Q^{\prime}$ by

$$
h(x)= \begin{cases}f(x) & \text { if } x \in P, \\ g(x) & \text { if } x \in Q\end{cases}
$$

Lemma 47. The map h from Definition 46 is well-defined and a subsumption map.

Proof. By Lemma 36 we may assume that $T_{P}=S_{Q}$ and $T_{P^{\prime}}=S_{Q^{\prime}}$, showing that $h$ is well-defined and a bijection. The other properties follow easily.

Lemma 48. If $P \sqsubseteq P^{\prime}$ and $Q \sqsubseteq Q^{\prime}$, then $P * Q$ is defined iff $P^{\prime} * Q^{\prime}$ is, and in that case, $P * Q \sqsubseteq$ $P^{\prime} * Q^{\prime}$.

Proof. Let $f: P \rightarrow P^{\prime}$ and $g: Q \rightarrow Q^{\prime}$ be the subsumption maps. The first claim is clear as $f$ and $g$ respect interfaces and labels. The second claim follows from Definition 46.

Using subsumption maps as 2-morphisms, ipomsets assemble as morphisms into a bicategory. Below, the identity on a linear pomset $(S,--\rightarrow, \lambda)$ is the discrete ipomset $\mathrm{id}_{S}={ }_{S} S_{S}=$ $(S, \emptyset,-\rightarrow, \lambda, S, S)$, with trivial precedence order and all points in both interfaces.

Proposition 49. Ipomsets form a (large) bicategory iPoms with objects linear pomsets $(S,-\rightarrow, \lambda)$, ipomsets $(P,<,-\rightarrow, \lambda, S, T)$ as morphisms from $(S,-\rightarrow, \lambda)$ to $(T,-\rightarrow, \lambda)$ with gluing as composition and identities $\mathrm{id}_{S}$, subsumptions as 2-morphisms, and ipomset isomorphisms as 2-isomorphisms.

Proof. It is clear that subsumption maps compose associatively and are invertible precisely when they are ipomset isomorphisms. Gluing composition is associative up-to 2-isomorphism, and the ipomsets $i_{S}$ are on-the-nose units for $*$. The pentagon identity is trivially satisfied due to uniqueness of 2-isomorphisms.

Remark 50. The bicategory iPoms is large as its objects and morphisms form a proper class. However, given that any ipomset is uniquely isomorphic to one on points $[k]$ and with interfaces $[n]$ and $[m]$, for some $k, n, m \geq 0$, iPoms is equivalent to its skeleton, which is a small 2-category; hence iPoms is essentially small.

By Lemma 41, interval ipomsets form a sub-bicategory of iPoms which we will denote iiPoms.

\section{Tracks and their labels}

We are now ready to introduce tracks in precubical sets, which are our model of computations, i.e., sequences of cells connected at faces. We define labels of tracks as interval ipomsets and show, conversely, how interval ipomsets give rise to tracks.

\subsection{Tracks}

Let $X$ be an event consistent precubical set. For $x, y \in X$, we say that $x$ is an elementary lower face of $y$, denoted $x \triangleleft y$, if $x=\delta_{i}^{0} y$ for some $i$; $x$ is an elementary upper face of $y$, denoted $y \triangleright x$, if $x=\delta_{i}^{1} y$. The reflexive transitive closures of the relations $\triangleleft$ and $\triangleright$ are denoted $\triangleleft^{*}$ and $\triangleright^{*}$. 
We say that $x$ is a lower, resp. upper face of $y$ if $x \triangleleft^{*} y$, resp. $y \triangleright^{*} x$. This is equivalent to the condition that $x=\delta_{A}^{0, \ldots, 0} y$ for some (possibly empty) $A$, resp. $x=\delta_{A}^{1, \ldots, 1} y$. By Lemma $22, A$ is determined uniquely by $x$ and $y$.

Definition 51. $A$ track in $X$ is a non-empty sequence $\rho=\left(x_{1}, \ldots, x_{m}\right), m \geq 1$, of elements of $X$ such that for all $i=1, \ldots, m-1, x_{i} \triangleleft^{*} x_{i+1}$ or $x_{i+1} \triangleright^{*} x_{i}$. A track $\rho$ as above is from $x_{1}$ to $x_{m}$, denoted $\rho: x_{1} \rightsquigarrow x_{m}$.

We allow repeated cells $x_{i}=x_{i+1}$ in tracks for notational convenience. A track $\left(x_{1}, \ldots, x_{m}\right)$ is full if it does not contain such repeated cells and all face relations are elementary, that is, $x_{i} \triangleleft x_{i+1}$ or $x_{i} \triangleright x_{i+1}$ for all $i=1, \ldots, m-1$. Any track without repeated cells may be filled to a full track by inserting appropriate (not necessarily unique) cells. In [42], full tracks are called execution paths.

Example 52. Figure 8 in the introduction displays the track $\left(x_{1}, x_{2}, x_{3}, x_{4}, x_{5}, x_{6}\right)$. As $x_{3} \triangleleft^{3} x_{4}$, this track is not full; it may be filled by inserting appropriate faces of $x_{4}$ and $x_{6}$, for example

$$
\left(x_{1}, x_{2}, x_{3}, \delta_{1}^{0} \delta_{1}^{0} x_{4}, \delta_{1}^{0} x_{4}, x_{4}, \delta_{1}^{1} x_{4}, x_{5}, x_{6}\right) .
$$

Definition 53. Let $\rho=\left(x_{1}, \ldots, x_{m}\right), \tau=\left(y_{1}, \ldots, y_{k}\right)$ be tracks in $X$. The concatenation $\rho * \tau$ of $\rho$ and $\tau$ is defined if $x_{m}=y_{1}$, and in that case, $\rho * \tau=\left(x_{1}, \ldots, x_{m}, y_{2}, \ldots, y_{k}\right)$.

The unit tracks are $(x)$ for $x \in X$. Tracks containing exactly two cells are called basic. Concatenation is associative, and every non-unit track is a unique concatenation of basic tracks. The following is clear.

Lemma 54. Tracks in $X$ form a small category Track $(X)$ with objects $x \in X$, tracks $\rho: x \rightsquigarrow y$ as morphisms, $*$ as composition, and identities $\mathrm{id}_{x}=(x)$.

\subsection{Labels of Tracks}

Let $(X, \lambda)$ be a labeled precubical set.

Definition 55. The label of a track $\rho$ in $X$ is the ipomset $\ell(\rho)$ defined recursively as follows:

- If $\rho=(x)$ is a unit track, then $\ell(\rho)=\mathrm{id}_{\ell(x)}$ : the identity ipomset on $\ell(x)$.

- If $\rho=(x, y)$ with $x \triangleleft^{*} y$, then $\ell(\rho)=\ell(x) \ell(y)_{\ell(y)}$ : a starter.

- If $\rho=(y, x)$ with $y \triangleright^{*} x$, then $\ell(\rho)=\ell(y) \ell(y)_{\ell(x)}$ : a terminator.

- If $\rho=\tau * \rho^{\prime}$ with $\tau$ a basic track, then $\ell(\rho)=\ell(\tau) * \ell\left(\rho^{\prime}\right)$.

By Proposition 44, labels of tracks are interval ipomsets, and $\ell\left(\rho_{1} * \rho_{2}\right) \cong \ell\left(\rho_{1}\right) * \ell\left(\rho_{2}\right)$ for all tracks $\rho_{1}, \rho_{2}$. The following is therefore clear.

Proposition 56. Labeling defines a functor $\ell: \operatorname{Track}(X) \rightarrow$ iiPoms.

Next we see that filling a track with extra cells does not change its label. Let $\sim$ be the equivalence on sets of tracks generated by $\left(x_{1}, \ldots, x_{m}\right) \sim\left(x_{1}, \ldots, x_{i-1}, x_{i+1}, \ldots, x_{m}\right)$ for $x_{i-1} \triangleleft^{*} x_{i} \triangleleft^{*}$ $x_{i+1}$ or $x_{i-1} \triangleright^{*} x_{i} \triangleright^{*} x_{i+1}$.

Lemma 57. If $\rho \sim \tau$, then $\ell(\rho) \cong \ell(\tau)$. 


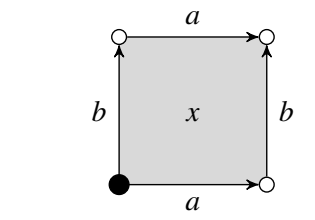

$\ell\left(\left(\delta_{1}^{0} \delta_{1}^{0} x, x\right)\right)=\left(\begin{array}{c}a \\ \vdots \\ b\end{array}\right)$

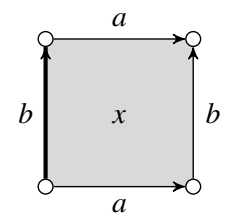

$\ell\left(\left(\delta_{1}^{0} x, x\right)\right)=\left(\begin{array}{c}a \\ \vdots \\ \breve{b}\end{array}\right)$,

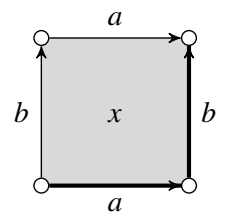

$\ell\left(\left(\delta_{2}^{0} x, x, \delta_{1}^{1} x\right)\right)=\stackrel{\left(\begin{array}{c}a \\ \vdots \\ \stackrel{b}{b}\end{array}\right)}{\longrightarrow}$

Figure 13. Some tracks and their labels in $X=\square^{\{a-\rightarrow b\}}$ (solid cells indicate the respective faces).

Proof. If $x \triangleleft^{*} y \triangleleft^{*} z$, then

$$
\ell((x, y, z))=\ell((x, y)) * \ell((y, z))={ }_{\ell(x)} \ell(y)_{\ell(y)} *_{\ell(y)} \ell(z)_{\ell(z)}={ }_{\ell(x)} \ell(z)_{\ell(z)}=\ell((x, z))
$$

from Lemma 43. The computations for $x \triangleright^{*} y \triangleright^{*} z$ are similar, and the result then follows by induction.

The next lemma shows that labels of tracks that consist of a cell and two of its faces on either side are discrete ipomsets. Its proof is a straightforward application of the definition.

Lemma 58. If $x \triangleleft^{*} y \triangleright^{*} z$, then $\ell((x, y, z))=\ell(x) \ell(y)_{\ell(z)}$.

Figure 13 shows some examples of simple tracks and their labels.

\subsection{Interval Ipomsets as Tracks}

We have seen how labels of tracks in HDAs can be computed as interval ipomsets. Now we show the inverse: how interval ipomsets may be converted into HDAs consisting essentially of a single track. To this end, first introduce a relation $\prec$ on the set $\{0,\ulcorner, 1\}$ by

$$
\prec=\{(0,0),(\ulcorner, 0),(1,0),(1,\ulcorner),(1,1)\} .
$$

The relation $\prec$ (which is not a partial order, given that it is neither reflexive nor irreflexive) corresponds to the meaning that we associate to the elements of $\{0,\ulcorner, 1\}: 0$ meaning the event has not yet started; $\ulcorner$ for an executing event; and 1 if the event has terminated. The intuition is that when $x$ and $y$ are events so that $x<y$ in the precedence order, then either $y$ has not yet started, in which case $x$ may be in any state, hence the first three pairs $(0,0),(\ulcorner, 0),(1,0)$; or $x$ has terminated and $y$ may be in any state, hence the last three pairs $(1,0),(1,\ulcorner),(1,1)$. In particular, it is impossible that both are active, so that $(\ulcorner,\ulcorner) \notin \prec$.

Remark 59. In Chu spaces for concurrency [35], $K_{3}=\left\{0<_{\mathrm{C}}\left\ulcorner<_{\mathrm{C}} 1\right\}\right.$ is the structure that defines the possible execution forms that an event can take. The intuition of the order $<_{\mathrm{C}}$ is that 0 (the event has not yet started) can happen only before $\lrcorner$ (the event is executing), which can happen only before 1 (the event has terminated). This order can be extended to sets of (execution values of) events, which in Chu terminology is called a state. For states, the order $<_{\mathrm{C}}$ expresses admissible sequences of executions of the system. For two independent events $x, y$, all execution forms $\left(e_{x}, e_{y}\right)$ with $e_{x}, e_{y} \in\{0,\ulcorner, 1\}$ would thus be admissible; but if there is a precendence order $x<y$, then the allowed tuples are precisely the ones in the relation $\prec$ above.

The following generalizes Definition 11 of the standard $S$-cube $\square^{S}$ on a linear poset $S$ to arbitrary ipomsets. 
Definition 60. For an ipomset $P$, define the $H D A\left(\square^{P}, I_{\square^{P}}, F_{\square^{P}}, \lambda_{\square^{P}}\right)$ as follows:

- $\square_{k}^{P}$ is the set of all relation-preserving functions $\left.x:\left(P,<_{P}\right) \rightarrow(\{0\lrcorner, 1\},, \prec\right)$ taking value \ulcorner on exactly $k$ elements;

- for $x \in \square_{k}^{P}$ and $x^{-1}\left(\ulcorner)=\left\{p_{1-\rightarrow P} \cdots-\rightarrow p_{P} p_{k}\right\}\right.$,

$$
\delta_{i}^{v}(x)(p)= \begin{cases}v & \text { for } p=p_{i} \\ x(p) & \text { for } p \neq p_{i}\end{cases}
$$

- $I_{\square^{P}}=\left\{i_{\square^{P}}\right\}$ and $F_{\square^{P}}=\left\{f_{\square^{P}}\right\}$ are given by

$$
i_{\square^{P}}(p)=\left\{\begin{array}{ll}
\ulcorner & \text { if } p \in S_{P}, \\
0 & \text { if } p \notin S_{P},
\end{array} f_{\square^{P}}(p)= \begin{cases}\ulcorner & \text { if } p \in T_{P}, \\
1 & \text { if } p \notin T_{P} ;\end{cases}\right.
$$

- For $x \in \square_{k}^{P}$ with $x^{-1}\left(\ulcorner)=\left\{p_{1-\rightarrow P} \cdots \rightarrow \rightarrow_{P} p_{k}\right\}, \lambda_{\square^{P}}(x)=\left(\lambda_{P}\left(p_{1}\right), \ldots, \lambda_{P}\left(p_{k}\right)\right)\right.$.

Above, $x^{-1}(\ulcorner)$ is indeed an antichain in the precedence order and hence linearly ordered by $-\rightarrow P$.

Example 61. Let $P$ be the ipomset

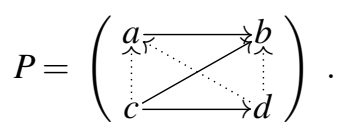

The cells of $\square^{P}$ are as follows, in increasing order of dimension (and with the event order omitted):

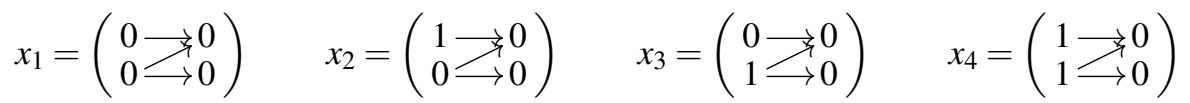

$$
\begin{aligned}
& x_{5}=\left(\begin{array}{l}
0 \rightrightarrows 0 \\
1 \rightrightarrows 1
\end{array}\right) \quad x_{6}=\left(\begin{array}{l}
1 \rightrightarrows 0 \\
1 \rightrightarrows 1
\end{array}\right) \quad x_{7}=\left(\begin{array}{l}
1 \rightrightarrows 1 \\
1 \rightrightarrows 0
\end{array}\right) \quad x_{8}=\left(\begin{array}{l}
1 \rightrightarrows 1 \\
1 \rightrightarrows 1
\end{array}\right)
\end{aligned}
$$

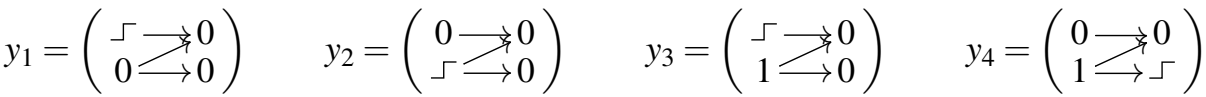

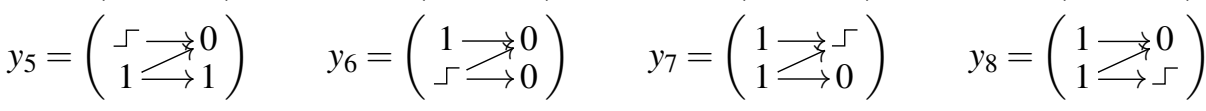

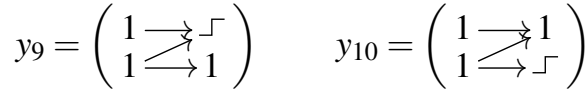

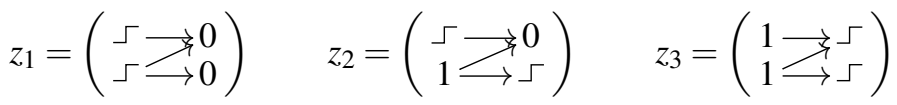

The cells $y_{1}, y_{3}$ and $y_{5}$ are labeled by $a, y_{7}$ and $y_{9}$ by $b, y_{2}$ and $y_{6}$ by $c, y_{4}, y_{8}, y_{10}$ by $d$. The labels of $z_{1}, z_{2}$ and $z_{3}$ are $(c, a),(d, a)$ and $(d, b)$, respectively. The order of letters in these pairs is determined be the event order on $P$. Geometrically these are arranged as shown in Figure 14 (left).

We will later apply Definition 60 to interval ipomsets to conclude in Proposition 92 that the language of $\square^{P}$ is generated by $P$. Our definition applies to general ipomsets, but as we will see, Proposition 92 fails for ipomsets which are not interval. It is an interesting open problem to characterize those HDA which are isomorphic to some $\square^{P}$. 

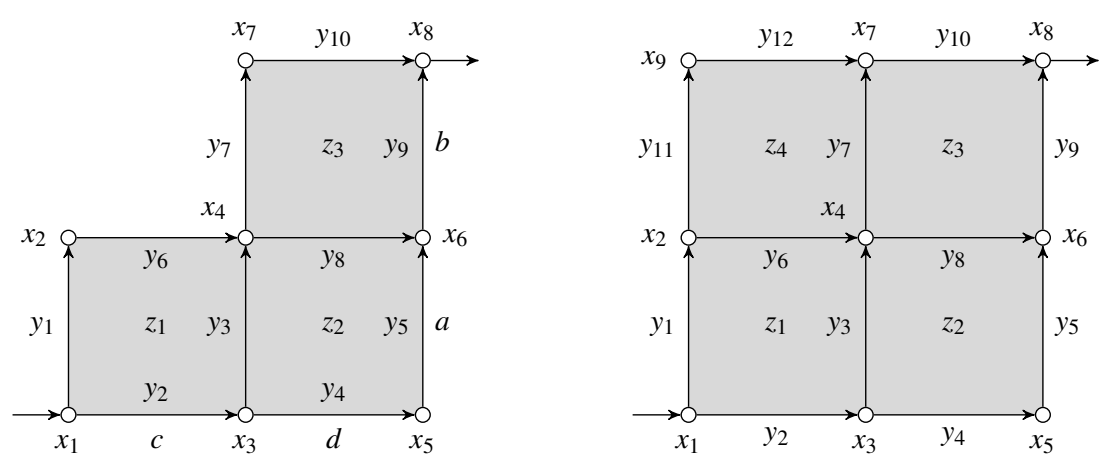

Figure 14. The HDAs $\square^{P}$ (left) and $\square^{Q}$ (right) from Examples 61 and 62 .
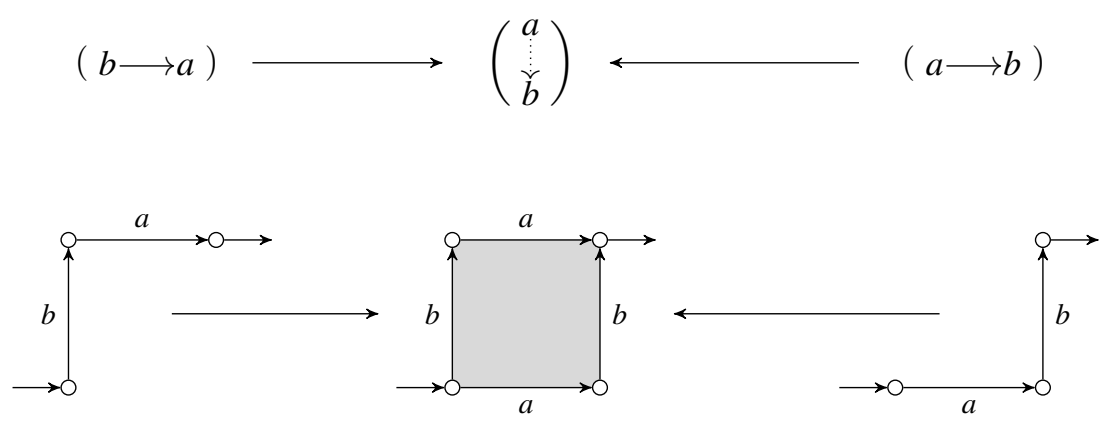

Figure 15. Subsumptions (top) give rise to HDA inclusions (bottom)

Example 62. If we instead of the ipomset $P$ of Example 61 take $Q$ to be the $2+2$-ipomset

$$
Q=\left(\begin{array}{c}
a \longrightarrow b \\
\stackrel{b}{\longrightarrow} d \\
c \longrightarrow d
\end{array}\right),
$$

then $\square^{Q}$ contains $\square^{P}$ and the following extra cells:

$$
x_{9}=\left(\begin{array}{l}
1 \longrightarrow 1 \\
0 \longrightarrow 0
\end{array}\right) \quad y_{11}=\left(\begin{array}{c}
1 \longrightarrow\ulcorner \\
0 \longrightarrow 0
\end{array}\right) \quad y_{12}=\left(\begin{array}{c}
1 \longrightarrow 1 \\
\ulcorner\longrightarrow 0
\end{array}\right) \quad z_{4}=\left(\begin{array}{c}
1 \longrightarrow 5 \\
\ulcorner\longrightarrow 0
\end{array}\right)
$$

Geometrically this amounts to adding the top-left square to $\square^{P}$, see Figure 14 (right).

The following can be shown by easy calculations; Figure 15 shows some simple examples.

Lemma 63. If $f: P \rightarrow Q$ is a subsumption map, then the function $\square^{f}: \square^{P} \rightarrow \square^{Q}$ given by $\square^{f}(x)(p)=x(f(p))$ is an injective HDA map.

For the next lemma, recall the notions of event consistency and universal events from Section 3.3.

Lemma 64. Let $P$ be an ipomset. Then $\square^{P}$ is event consistent, and $E_{\square^{P}} \cong P$ as labeled sets. For every $x \in \square^{P}, \operatorname{ev}(x)=x^{-1}(\ulcorner)$ as linear posets. 

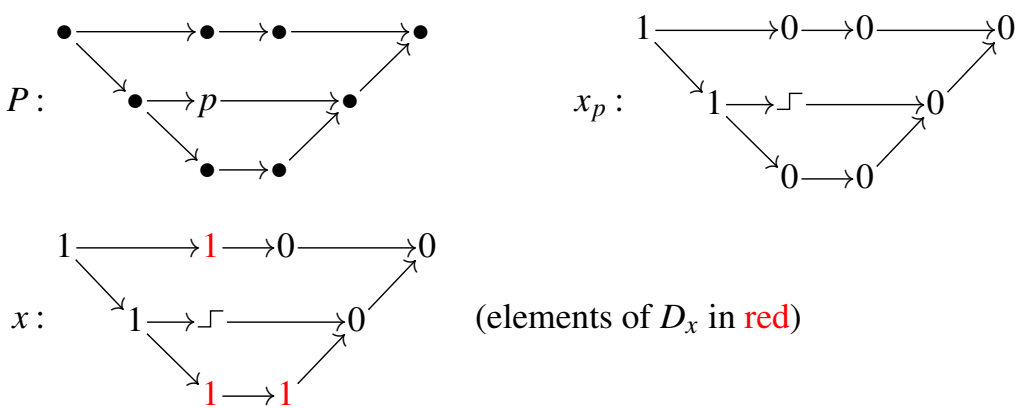

(elements of $D_{x}$ in red)
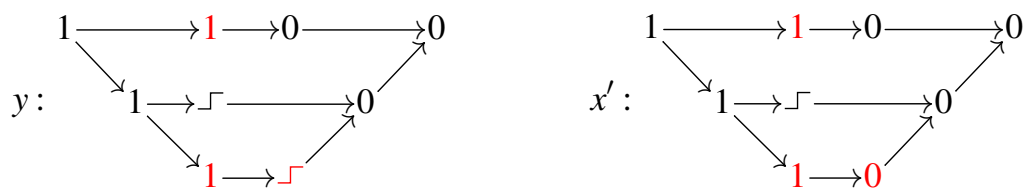

Figure 16. Pomsets and cells in the proof of Lemma 64.

Proof. To show that $\square^{P}$ is event consistent, let $f: P \rightarrow Q$ be a subsumption map into a discrete ipomset $Q ; Q$ may be obtained from any linearization of $-\rightarrow P$. Then the precubical set underlying $\square^{Q}$ is a standard cube, and by Lemma 63, $\square^{f}: \square^{P} \rightarrow \square^{Q}$ is an embedding. By Example 19, $\square^{P}$ is event consistent.

There is a $P$-labeling of $\square^{P}$, i.e., a precubical map $\square^{P} \rightarrow ! P$ that sends $x \in \square^{P}$ to $x^{-1}(\ulcorner)=$ $\left(p_{1}-\rightarrow_{P} \cdots \rightarrow \rightarrow_{P} p_{n}\right)$. This induces a function $\pi: E_{\square} \rightarrow P$ (Proposition 20). For every $p \in P$, define $x_{p} \in \square_{1}^{P}$ by

$$
x_{p}(q)= \begin{cases}1 & \text { if } q<_{P} p \\ \ulcorner & \text { if } q=p \\ 0 & \text { otherwise }\end{cases}
$$

Since $\pi\left(x_{p}\right)=p, \pi$ is surjective.

It remains to show that $\pi$ is injective. Let $x \in \square_{1}^{P}$ satisfy $\pi(x)=p$; we will show that $x \sim_{\mathrm{ev}} x_{p}$. Note that $x(q)=\ulcorner$ iff $q=p$. We proceed by induction on the number of elements in the set $D_{x}=\left\{q \in P \mid x(q) \neq x_{p}(q)\right\}$, see Figure 16 for an illustration.

All elements of $D_{x}$ are parallel with $p$, since monotonicity of $x$ and $x_{p}$ implies that $x(q)=$ $x_{p}(q)=1$ if $q<_{P} p$ and $x(q)=x_{p}(q)=0$ if $p<_{P} q$. Thus, $x_{p}(q)=0$ and $x(q)=1$ for all $q \in D_{x}$.

Let $q \in D_{x}$ be a $<_{P}$-maximal element. Let $y: P \rightarrow\{0,\ulcorner, 1\}$ be given by $y(q)=\ulcorner$ and $y(r)=$ $x(r)$ for $r \neq q$. We show that $y$ is monotone and hence a 2-cell in $\square^{P}$.

We have $y(r)=x(r)=1$, hence $y(r) \prec y(q)$, for all $r<_{P} q$, since $x$ preserves $<_{P}$. For $q<_{P} r$, on the other hand, maximality of $q$ in $D_{x}$ implies that $r \notin D_{x}$, so that $y(r)=x(r)=x_{p}(r)=0$, hence $y(q) \prec y(r)$. Given that $y(r)=x(r)$ for $r \neq q$, we have shown that $y$ is monotone.

Now $y(p)=y(q)=\left\ulcorner\right.$, so $y$ is a 2-cell in $\square^{P}$. Let $i=1$ if $q-\rightarrow p$ and $i=2$ if $p \rightarrow q$, then $\delta_{i}^{1}(y)=x$. Let $x^{\prime}=\delta_{i}^{0}(y)$, then also $\pi\left(x^{\prime}\right)=p$, and $D_{x^{\prime}}=D_{x} \backslash\{q\}$. The inductive hypothesis asserts that $x=\delta_{i}^{1}(y) \sim{ }_{\mathrm{ev}} \delta_{i}^{0}(y)=x^{\prime} \sim_{\mathrm{ev}} x_{p}$.

Next we see that gluings of ipomsets correspond to pushouts of their HDA objects. Recall the Yoneda inclusions $\mathbf{i}_{x}$ from Lemma 12. 
Lemma 65. Let $Q$ and $R$ be composable ipomsets with $T_{Q} \cong S \cong S_{R}$ and $P=Q * R$. There is a pushout

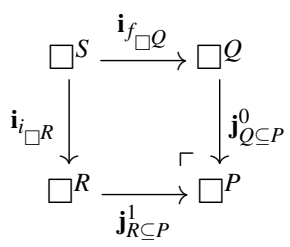

where

$$
\mathbf{j}_{Q \subseteq P}^{0}(x)(p)=\left\{\begin{array}{ll}
x(p) & \text { for } p \in Q, \\
0 & \text { otherwise, }
\end{array} \quad \mathbf{j}_{R \subseteq P}^{1}(x)(p)= \begin{cases}x(p) & \text { for } p \in R \\
1 & \text { otherwise }\end{cases}\right.
$$

Proof. It is clear that all the maps in the diagram are injective. Fix $x \in \square^{P}$.

- If there exists $q \in Q \backslash T_{Q}$ with $x(q) \in\left\{0,\ulcorner\}\right.$, then obviously $x \notin \mathbf{j}_{R \subseteq P}^{1}\left(\square^{R}\right)$. But for all $r \in$ $R \backslash S_{R}$ we have $q<r$ and then $x(r)=0$. It is easy to verify that the restriction $x_{\mid Q} \in \square^{Q}$ and then $x=\mathbf{j}_{Q \subseteq P}^{0}\left(x_{\mid Q}\right) \in \mathbf{j}_{Q \subseteq P}^{0}\left(\square^{Q}\right)$.

- Similarly, if $x(r) \in\left\{\ulcorner, 1\}\right.$ for some $r \in R \backslash S_{R}$, then $x \in \mathbf{j}_{R \subseteq P}^{1}\left(\square^{R}\right)$.

- We have

$$
\mathbf{j}_{Q \subseteq P}^{0} \circ \mathbf{i}_{f_{\square Q}}(y)(p)=\mathbf{j}_{R \subseteq P}^{1} \circ \mathbf{i}_{i_{\square}}(y)(p)= \begin{cases}1 & \text { for } p \in Q \backslash T_{Q}, \\ \ulcorner & \text { for } p \in S \cong T_{Q} \cong S_{R}, \\ 0 & \text { for } p \in R \backslash S_{R} .\end{cases}
$$

Thus, the diagram commutes. Denote $j=\mathbf{j}_{Q \subseteq P}^{0} \circ \mathbf{i}_{f_{\square Q}}=\mathbf{j}_{R \subseteq P}^{1} \circ \mathbf{i}_{i_{\square}}$. The condition $x(q)=1$ for all $q \in Q \backslash T_{Q}$ and $x(r)=0$ for all $r \in R \backslash S_{R}$ is equivalent to both $x \in \mathbf{j}_{Q \subseteq P}^{0}(\square Q) \cup$ $\mathbf{j}_{R \subseteq P}^{1}\left(\square^{R}\right)$ and $x \in j\left(\square^{P}\right)$.

As a consequence, $\square^{P}=\mathbf{j}_{Q \subseteq P}^{0}\left(\square^{Q}\right) \cup \mathbf{j}_{R \subseteq P}^{1}\left(\square^{R}\right)$ and $j\left(\square^{S}\right)=\mathbf{j}_{Q \subseteq P}^{0}\left(\square^{Q}\right) \cap \mathbf{j}_{R \subseteq P}^{1}\left(\square^{R}\right)$.

Lemma 66. Let $X$ be a labeled precubical set, $\rho: x \rightsquigarrow y \in \operatorname{Track}(X)$, and $P=\ell(\rho)$. There is a map of labeled precubical sets $g: \square^{P} \rightarrow X$ such that $g\left(i_{\square^{P}}\right)=x$ and $g\left(f_{\square^{P}}\right)=y$.

Proof. Induction on the number of cells in $\rho$.

- If $\rho=(x)$, then $P=\mathrm{id}_{\ell(x)}$ and $\square^{P}=\square^{\mathrm{ev}(x)}$. The Yoneda map $\mathbf{i}_{x}: \square^{\mathrm{ev}(x)} \rightarrow X$ satisfies the required condition.

- If $\rho=(x, y)$ with $x \triangleleft^{*} y$, then $P=\ell(x) \ell(y)_{\ell(y)}$. Again, we may take $g=\mathbf{i}_{y}$.

- The case $\rho=(y, x)$ with $y \triangleright^{*} x$ is similar.

- In case $\rho=\sigma * \tau$, where both $\sigma: x \rightsquigarrow z$ and $\tau: z \rightsquigarrow y$ are shorter than $\rho$, let $Q=\ell(\sigma), R=$ $\ell(\tau)$. By the inductive hypothesis, there are labeled precubical maps $g_{Q}: \square^{Q} \rightarrow X$ and $g_{R}$ : $\square^{R} \rightarrow X$ such that $g_{Q}\left(i_{\square Q}\right)=x, g_{R}\left(f_{\square R}\right)=y$, and $g_{Q}\left(f_{\square Q}\right)=g_{R}\left(i_{\square^{R}}\right)=z$. The last equality, together with Lemma 65, guarantees that $g_{Q}$ and $g_{R}$ glue to a map $g: \square^{P} \rightarrow X$. It is clear that $g\left(i_{\square^{P}}\right)=g_{Q}\left(i_{\square Q}\right)=x$ and $g\left(f_{\square^{P}}\right)=g_{R}\left(f_{\square^{R}}\right)=y$.

Proposition 67. For any interval ipomset $P$ there exists a track $\rho: i_{\square^{P}} \rightsquigarrow f_{\square^{P}}$ such that $\ell(\rho) \cong P$. 
Proof. If $P={ }_{S} U_{T}$ is discrete, then $\ell\left(\left(i_{\square^{P}}, \mathbf{y}_{U}, f_{\square^{P}}\right)\right) \cong P$. If $P$ is not discrete, then there is a presentation $P=Q * R$ (Proposition 44). If $\sigma: i_{\square Q} \rightsquigarrow f_{\square Q}$ is a track with $\ell(\sigma) \cong Q$ and $\tau: i_{\square R} \rightsquigarrow f_{\square R}$ a track with $\ell(\tau) \cong R$, then

$$
\ell\left(\mathbf{j}_{Q \subseteq P}^{0}(\sigma) * \mathbf{j}_{R \subseteq P}^{1}(\tau)\right) \cong Q * R=P
$$

by Lemma 65.

Example 68. We follow up on Example 61. For

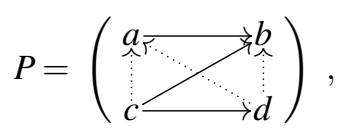

we have $i_{\square}=x_{1}$ and $f_{\square}=x_{8}$ (see also Figure 14), and the track $\rho$ of the proposition is given by $\rho=\left(x_{1}, z_{1}, y_{3}, z_{2}, y_{8}, z_{3}, x_{8}\right)$. If we add interfaces to $P$, for example

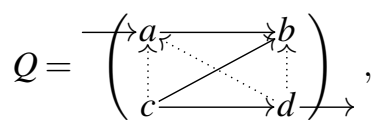

then $i_{\square Q}=y_{1}, f_{\square Q}=y_{10}$, and $\rho=\left(y_{1}, z_{1}, y_{3}, z_{2}, y_{8}, z_{3}, y_{10}\right)$.

\section{The Geometric View}

Precubical sets may be realized as directed topological spaces, and then directed paths through these spaces give an intuitive model of computations. In this section we first recap the geometric realization and then introduce labels of directed paths in HDAs. We will see that for every directed path there exists a track with the same label, and vice versa, so that HDA languages defined using tracks and using directed paths are the same.

\subsection{Geometric Realization}

Recall that the concatenation $\alpha * \beta$ of two paths $\alpha, \beta: I=[0,1] \rightarrow \mathscr{X}$ in a topological space $\mathscr{X}$ is defined, if $\alpha(1)=\beta(0)$, as

$$
\alpha * \beta(t)= \begin{cases}\alpha(2 t) & \text { for } t \leq \frac{1}{2} \\ \beta(2 t-1) & \text { for } t \geq \frac{1}{2} .\end{cases}
$$

A directed topological space, or $d$-space [19] is a pair $(\mathscr{X}, \vec{P} \mathscr{X})$ consisting of a topological space $\mathscr{X}$ and a set $\vec{P} \mathscr{X} \subseteq \mathscr{X}^{I}$ of paths in $\mathscr{X}$ such that $\vec{P} \mathscr{X}$

- contains all constant paths;

- is closed under concatenation: if $\alpha, \beta \in \vec{P} \mathscr{X}$ and $\alpha(1)=\beta(0)$, then $\alpha * \beta \in \vec{P} \mathscr{X}$;

- is closed under reparametrization and subpath: for any $\alpha \in \vec{P} \mathscr{X}$ and $h: I \rightarrow I$ continuous and (weakly) increasing, also $\alpha \circ h \in \vec{P} \mathscr{X}$.

The elements of $\vec{P} \mathscr{X}$ are called directed paths or d-paths.

Prominent examples of d-spaces are the directed interval $\vec{I}=[0,1]$ with the natural ordering on the real numbers and the directed $n$-cubes $\vec{I}^{n}$ for $n \geq 0$. Similarly, there are directed Euclidean spaces $\overrightarrow{\mathbb{R}}^{n}$ for all $n \geq 0$. In each of these, the d-paths a precisely the paths which are (weakly) increasing in each coordinate, that is, $\alpha: I \rightarrow \overrightarrow{\mathbb{R}}^{n}$ is a d-path iff $t_{1} \leq t_{2}$ implies $\alpha\left(t_{1}\right) \leq \alpha\left(t_{2}\right)$ in the usual ordering $\left(x_{1}, \ldots, x_{n}\right) \leq\left(y_{1}, \ldots, y_{n}\right)$ iff $x_{i} \leq y_{i}$ for all $i$. 
Morphisms $f:(\mathscr{X}, \vec{P} \mathscr{X}) \rightarrow(\mathscr{Y}, \vec{P} \mathscr{Y})$ of d-spaces are $d$-maps; they are those continuous functions that also preserve directedness, i.e., $f \circ \alpha \in \vec{P} \mathscr{Y}$ for all $\alpha \in \vec{P} \mathscr{X}$. For any d-space $(\mathscr{X}, \vec{P} \mathscr{X})$ we have $\vec{P} \mathscr{X}=\mathscr{X}^{\vec{I}}$ as function spaces.

The so-defined category dTop of d-spaces is complete and cocomplete [19]. In particular, quotients of d-spaces are well-defined. If $\mathscr{X}$ is a d-space and $\sim$ an equivalence on $\mathscr{X}$, then d-paths in the quotient space $\mathscr{X} / \sim$ are of the form

$$
\alpha=\left(\pi\left(\beta_{1}\right) * \cdots * \pi\left(\beta_{m}\right)\right) \circ h
$$

where all $\beta_{i}$ are d-paths in $\mathscr{X}$ such that $\beta_{i}(1) \sim \beta_{i+1}(0)$ and $h: \vec{I} \rightarrow \vec{I}$ is a surjective d-map.

Surjective d-maps $h: \vec{I} \rightarrow \vec{I}$ as above are called reparametrizations and will play a central role below.

Definition 69. The geometric realization of a precubical set $X$ is the $d$-space

$$
|X|=\bigsqcup_{n \geq 0} X_{n} \times \vec{I}^{n} / \sim,
$$

where the equivalence relation $\sim$ is generated by

$$
\left(\delta_{i}^{v} x,\left(t_{1}, \ldots, t_{n-1}\right)\right) \sim\left(x,\left(t_{1}, \ldots, t_{i-1}, v, t_{i+1}, \ldots, t_{n-1}\right)\right) .
$$

The geometric realization of a precubical map $f: X \rightarrow Y$ is the d-map $|f|:|X| \rightarrow|Y|$ given by $|f|\left(\left[x,\left(t_{1}, \ldots, t_{n}\right)\right]\right)=\left[f(x),\left(t_{1}, \ldots, t_{n}\right)\right]$.

Above, $\left[x,\left(t_{1}, \ldots, t_{n}\right)\right]$ is used to denote equivalence classes of $\sim$. Geometric realization is a functor from Set ${ }^{\circ \text { op }}$ to dTop.

Example 70. The geometric realization of the $n$-cube $\square^{n}$ is the directed cube $\vec{I}^{n}$. The purpose of the equivalence relation $\sim$ in the definition is to embed faces as subspaces, for example, the elementary face $\delta_{1}^{0} \mathbf{y}_{n}$ of the top cell of $\square^{n}$ is the subset $\left\{\left(0, t_{2}, \ldots, t_{n}\right) \mid 0 \leq t_{i} \leq 1\right\} \subseteq I^{n}$.

The interior image $] x\left[\subseteq|X|\right.$ of a cell $x \in X_{n}$ in a precubical set $X$ is defined as

$$
] x\left[=\left\{\left[x,\left(t_{1}, \ldots, t_{n}\right)\right] \mid 0<t_{i}<1 \text { for all } i \in[n]\right\} .\right.
$$

The set $] x\left[\right.$ is open for $x \notin X_{0}$; for $\left.x \in X_{0},\right] x[=\{x\}$.

Definition 71. The carrier $\operatorname{carr}(p)$ of a point $p \in|X|$ is the unique cell $x \in X$ such that $p \in] x[$.

For later use we record the following lemma, whose proof easily follows from the definition; see also [7]:

Lemma 72. For a precubical map $f: X \rightarrow Y$ and $p \in|X|, \operatorname{carr}(|f|(p))=f(\operatorname{carr}(p))$.

We conclude with a description of d-paths on $|X|$. Recall the Yoneda inclusions $\mathbf{i}_{x}: \square^{n} \rightarrow X$ from Lemma 12. These induce d-maps $\left|\mathbf{i}_{x}\right|: \vec{I}^{n} \rightarrow|X|$.

Lemma 73. Every d-path $\alpha \in \vec{P}|X|$ has a presentation

$$
\alpha=\left(\left(\left|\mathbf{i}_{x_{1}}\right| \circ \beta_{1}\right) *\left(\left|\mathbf{i}_{x_{2}}\right| \circ \beta_{2}\right) * \cdots *\left(\left|\mathbf{i}_{x_{m}}\right| \circ \beta_{m}\right)\right) \circ h,
$$

where $x_{1}, \ldots, x_{m} \in X, \quad \beta_{i} \in \vec{P}\left(\vec{I}^{\mathrm{dim} x_{i}}\right), \quad\left[x_{i}, \beta_{i}(1)\right]=\left[x_{i+1}, \beta_{i+1}(0)\right] \in|X|$ and $h: \vec{I} \rightarrow \vec{I}$ is a reparametrization. Moreover, we can assume that $\operatorname{carr}\left(\beta_{i}\left(\frac{1}{2}\right)\right)=\mathbf{y}_{\operatorname{dim}\left(x_{i}\right)}$. 


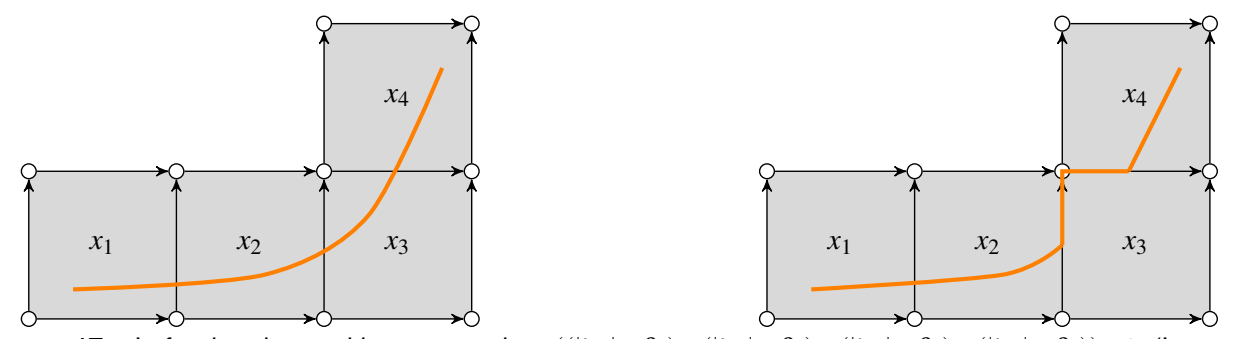

Figure 17. Left: d-path $\alpha$ with presentation $\left(\left(\left|\mathbf{i}_{x_{1}}\right| \circ \beta_{1}\right) *\left(\left|\mathbf{i}_{x_{2}}\right| \circ \beta_{2}\right) *\left(\left|\mathbf{i}_{x_{3}}\right| \circ \beta_{3}\right) *\left(\left|\mathbf{i}_{x_{4}}\right| \circ \beta_{4}\right)\right) \circ h$ (Lemma 73); right: counterexample in the proof of Lemma 73, with presentation $\left(\left(\left|\mathbf{i}_{x_{1}}\right| \circ \beta_{1}\right) *\left(\left|\mathbf{i}_{x_{2}}\right| \circ \beta_{2}\right) *\left(\left|\mathbf{i}_{\delta_{1}^{0} x_{3}}\right| \circ \gamma_{1}\right) *\right.$ $\left.\left(\left|\mathbf{i}_{\delta_{1}^{1} x_{3}}\right| \circ \gamma_{2}\right) *\left(\left|\mathbf{i}_{x_{4}}\right| \circ \beta_{4}\right)\right) \circ h^{\prime \prime}$.

Figure 17 shows an example: on the left, a d-path and a presentation; on the right, the counterexample used below in the proof.

Proof. Apart from the last statement, this follows immediately from the description of d-paths on quotient d-spaces and the definition of the geometric realization.

Let $\mathfrak{S}$ be the set of sequences $\left(d_{0}, d_{1}, \ldots\right)$ of natural numbers which are eventually vanishing, that is, there exists $n \geq 0$ such that $d_{j}=0$ for all $j>n$. Equip $\mathfrak{S}$ with the reverse lexicographic order, i.e., $\left(d_{j}\right)<\left(d_{j}^{\prime}\right)$ if there exists $n$ such that $d_{n}<d_{n}^{\prime}$ and $d_{j}=d_{j}^{\prime}$ for $j>n$. For every presentation $(*)$ of $\alpha$ we associate the sequence $\left(d_{j}\right) \in \mathfrak{S}$ such that $d_{j}$ is the number of indices $i$ such that $\operatorname{dim}\left(x_{i}\right)=j$. Choose a presentation $(*)$ with a minimal associated sequence $\left(d_{j}\right) \in \mathfrak{S}$. Denote $n_{i}=\operatorname{dim} x_{i}$.

Assume that for some $\left.i, \beta_{i}(t) \notin\right] \mathbf{y}_{n_{i}}$ [ for all $t$. But then $\beta_{i} \in \vec{P}\left|\square_{n_{i}-1}^{n_{i}}\right|$, the set of d-paths in the $\left(n_{i}-1\right)$-restriction of $\square^{n_{i}}$, and hence it has a presentation

$$
\beta_{i}=\left(\left(\left|\mathbf{i}_{y_{1}}\right| \circ \gamma_{1}\right) * \cdots *\left(\left|\mathbf{i}_{y_{l}}\right| \circ \gamma_{l}\right)\right) \circ h^{\prime} .
$$

Obviously $\operatorname{dim}\left(y_{k}\right)<n_{i}$ for all $k$. Collecting these two presentations, we have

$$
\begin{aligned}
\alpha=\left(\left(\left|\mathbf{i}_{x_{1}}\right| \circ \beta_{1}\right) * \cdots *\right. & \left(\left|\mathbf{i}_{x_{i-1}}\right| \circ \beta_{i-1}\right) *\left(| \mathbf { i } _ { x _ { i } } | \circ \left(\left(\left|\mathbf{i}_{y_{1}}\right| \circ \gamma_{1}\right) * \cdots\right.\right. \\
& \left.\left.\left.\cdots *\left(\left|\mathbf{i}_{y_{l}}\right| \circ \gamma_{l}\right)\right) \circ h^{\prime}\right) *\left(\left|\mathbf{i}_{x_{i+1}}\right| \circ \beta_{i+1}\right) * \cdots *\left(\left|\mathbf{i}_{x_{m}}\right| \circ \beta_{m}\right)\right) \circ h \\
=\left(\left(\left|\mathbf{i}_{x_{1}}\right| \circ \beta_{1}\right) * \cdots *\right. & \left(\left|\mathbf{i}_{x_{i-1}}\right| \circ \beta_{i-1}\right) *\left(\mid \mathbf{i}_{\mathbf{i}_{x_{i}}}\left(y_{1}\right)\right. \\
\cdots & \left.\circ \gamma_{1}\right) * \cdots \\
\cdots *\left(\mid \mathbf{i}_{\mathbf{i}_{i}}\left(y_{l}\right)\right. & \left.\left.\circ \gamma_{l}\right) *\left(\left|\mathbf{i}_{x_{i+1}}\right| \circ \beta_{i+1}\right) * \cdots *\left(\left|\mathbf{i}_{x_{m}}\right| \circ \beta_{m}\right)\right) \circ h^{\prime \prime}
\end{aligned}
$$

for some reparametrization $h^{\prime \prime}$ obtained from $h$ and $h^{\prime}$. Let $\left(d_{j}^{\prime}\right)$ be the associated sequence of this presentation. Then $d_{j}^{\prime}=d_{j}$ for $j>n_{i}$ and $d_{n_{i}}^{\prime}=d_{n_{i}}-1$, since $x_{i}$ no longer appears in the presentation and cells $\mathbf{i}_{x_{i}}\left(y_{k}\right)$ have smaller dimensions: a contradiction to the minimality of $\left(d_{j}\right)$.

As a consequence, for every $i$ there exists $t_{i}$ with $\operatorname{carr}\left(\beta_{i}\left(t_{i}\right)\right)=\mathbf{y}_{n_{i}}$. By reparametrizing $\beta_{i}$ and adjusting $h^{\prime \prime}$ we can ensure that $t_{i}=\frac{1}{2}$.

\subsection{Labels of d-paths}

For the rest of this section, $(X, \lambda)$ is a labeled precubical set (which is, by definition, event consistent). We will associate to every d-path $\alpha$ in $|X|$ its label $\ell(\alpha)$ as an interval ipomset. In order to do so, we first need to find the (universal) events in $X$ that are active during the execution $\alpha$.

We say that an event $e \in E_{X}$ is active at point $p=\left[x,\left(t_{1}, \ldots, t_{n}\right)\right] \in|X|$, for $x \in X_{n}$, if there is $i \in[n]$ such that $\operatorname{ev}_{i}(x)=e$ and $0<t_{i}<1$. Otherwise, $e$ is inactive at $p$. It is easy to verify that this does not depend on the choice of a presentation of $p$. Let $U_{e}^{X} \subseteq|X|$ be the set of points in which $e$ is active. The following is clear. 


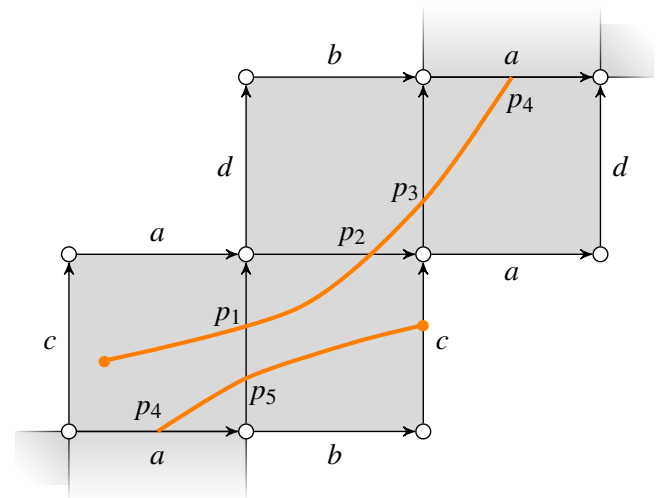

Figure 18. Directed path which wraps around a two-dimensional loop (bottom left and top right edges identified).

Lemma 74. $U_{e}^{X}=\bigcup\{] x[\mid e \in \operatorname{ev}(x)\}=\{p \in|X| \mid e \in \operatorname{ev}(\operatorname{carr}(p))\}$.

Note that all events are inactive at vertices, exactly one event is active along an edge, and so on: if $\operatorname{dim}(\operatorname{carr}(p))=n$, then exactly the $n$ events in $\operatorname{ev}(\operatorname{carr}(p))$ are active at $p$. We will write $U_{e}$ for $U_{e}^{X}$ when $X$ is clear.

Now fix a d-path $\alpha \in \vec{P}|X|$. For every event $e \in E_{X}$, let

$$
J_{e}^{\alpha}=\alpha^{-1}\left(U_{e}\right) \subseteq[0,1]
$$

be the set of points in time when $e$ is active. $J_{e}^{\alpha}$ is an open subset of $[0,1]$, since $U_{e}$ is open. Moreover, by Lemma 73 it has a finite number of connected components. Thus, there is a unique presentation

$$
J_{e}^{\alpha}=I_{e, 1}^{\alpha} \cup \cdots \cup I_{e, n_{e}^{\alpha}}^{\alpha}
$$

as a union of connected components ordered increasingly. Each of these components is open in $[0,1]$, though not necessarily in $\mathbb{R}$ : possibly $I_{e, 1}^{\alpha}=\left[0, t\left[\right.\right.$ or $\left.\left.T_{e, n_{e}^{\alpha}}^{\alpha}=\right] t, 1\right]$, or even $I_{e, 1}^{\alpha}=[0,1]$ for $n_{e}^{\alpha}=1$. The collection of presentations (3) is called the interval arrangement of $\alpha$.

Example 75. Figure 18 shows a d-path $\alpha$ through a labeled precubical set with a two-dimensional loop: $\alpha$ starts inside the bottom-left square with events $a$ and $c$, continues until the upper face of the top-right square, which is identified with the lower face of the bottom-left square, and finishes in the right $c$-labeled edge. Assuming that $\alpha$ is parametrized so that $\alpha\left(\frac{i}{6}\right)=p_{i}$ for $i \in[5]$ (the intersection points $p_{1}, \ldots, p_{5}$ of $\alpha$ with the edges are indicated in the figure), its interval arrangement is

$$
\left.J_{a}^{\alpha}=\left[0, \frac{1}{6}[\cup] \frac{1}{2}, \frac{5}{6}\right], \quad J_{b}^{\alpha}=\right] \frac{1}{6}, \frac{1}{2}[\cup] \frac{5}{6}, 1\left[, \quad J_{c}^{\alpha}=\left[0, \frac{1}{3}[\cup] \frac{2}{3}, 1\right], \quad J_{d}^{\alpha}=\right] \frac{1}{3}, \frac{2}{3}[.
$$

Now, for every $x \in X$, define a relation $\rightarrow_{x}$ on $\operatorname{ev}(x)$ by $e-\rightarrow_{x} e^{\prime}$ if $e=\operatorname{ev}_{i}(x)$ and $e^{\prime}=\mathrm{ev}_{j}(x)$ for $i<j$. From Lemma 26 we immediately get

Lemma 76. If $e, e^{\prime} \in \delta_{i}^{v} x$ for some $i$ and $v$, then $e-\rightarrow_{i}^{v} x e^{\prime}$ iff $e-\rightarrow_{x} e^{\prime}$.

As a consequence, on every connected component $C \subseteq U_{e} \cap U_{e^{\prime}}$ there is a well-defined relation $\rightarrow \rightarrow_{C}$ between $e$ and $e^{\prime}$ (although it may differ between different components). We write $\rightarrow \rightarrow_{p}=$ $\rightarrow C$ for any point $p \in C$.

Definition 77. The label of $\alpha$ is the ipomset $\ell(\alpha)=\left(P,<_{P},-\rightarrow_{P}, \lambda_{P}, S_{P}, T_{P}\right)$ given as follows: 


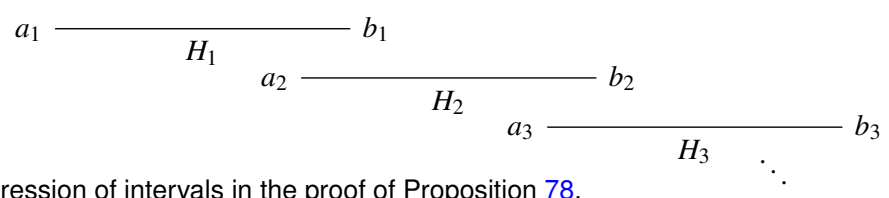

Figure 19. Progression of intervals in the proof of Proposition 78

- $P=\left\{(e, i) \mid e \in E_{X}, 1 \leq i \leq n_{e}^{\alpha}\right\}$

- $(e, i)<_{P}\left(e^{\prime}, i^{\prime}\right)$ if $I_{e, i}^{\alpha}<I_{e^{\prime}, i^{\prime}}^{\alpha}$;

$-\rightarrow P$ is the transitive closure of the relations $(e, i)-\rightarrow \alpha(t)\left(e^{\prime}, i^{\prime}\right)$ for $t \in I_{e, i}^{\alpha} \cap I_{e^{\prime}, i^{\prime}}^{\alpha}$ (this does not depend on the choice of $t$ since $I_{e, i}^{\alpha} \cap I_{e^{\prime}, i^{\prime}}^{\alpha}$ is connected);

- $\lambda_{P}((e, i))=\lambda^{\mathrm{ev}}(e), S_{P}=\left\{(e, i) \in P \mid 0 \in I_{e, i}^{\alpha}\right\}$, and $T_{P}=\left\{(e, i) \in P \mid 1 \in I_{e, i}^{\alpha}\right\}$.

Hence all elements of $S_{P}$ are of the form $(e, 1)$ and all elements of $T_{P}$ are of the form $\left(e, n_{e}^{\alpha}\right)$. Further, $S_{P} \cong \operatorname{carr}(\alpha(0))$ and $T_{P} \cong \operatorname{carr}(\alpha(1))$ as linear posets.

Proposition 78. The label $\ell(\alpha)$ is an interval ipomset.

Proof. By definition, $<_{P}$ is an interval order, $S_{P}$ contains only $<_{P}$-minimal elements, and $T_{P}$ contains only $<_{P}$-maximal elements. Assume that $(e, i)$ and $\left(e^{\prime}, i^{\prime}\right)$ are $<_{P}$-incomparable, then $I_{e, i}^{\alpha} \cap I_{e^{\prime}, i^{\prime}}^{\alpha} \neq \emptyset$. Let $t \in I_{e, i}^{\alpha} \cap I_{e^{\prime}, i^{\prime}}^{\alpha}$, then $(e, i) \rightarrow_{\alpha(t)}\left(e^{\prime}, i^{\prime}\right)$ or $\left(e^{\prime}, i^{\prime}\right)-\rightarrow_{\alpha(t)}(e, i)$, hence $(e, i)$ and $\left(e^{\prime}, i^{\prime}\right)$ are $\rightarrow P^{- \text {comparable. }}$

It remains to show that $\rightarrow \rightarrow P$ is irreflexive. So let

$$
\left(e_{1}, i_{1}\right)-\rightarrow \alpha\left(t_{1}\right) \cdots-\rightarrow \alpha\left(t_{r-1}\right)\left(e_{r}, i_{r}\right) \rightarrow \alpha\left(t_{r}\right)\left(e_{1}, i_{1}\right)
$$

be a shortest loop of elementary relations and denote $\left.H_{k}=I_{e_{k}, i_{k}}^{\alpha}=\right] a_{k}, b_{k}\left[\right.$ (or $\left[a_{k}, b_{k}[,] a_{k}, b_{k}\right]$, or $\left[a_{k}, b_{k}\right]$, in case $a_{k}=0$ or $b_{k}=1$; this will not matter for our argument below).

We have $H_{k} \cap H_{k+1} \neq \emptyset$ for $k \in[r-1]$, and also $H_{r} \cap H_{1} \neq \emptyset$. On the other hand, $H_{k} \cap H_{l}=\emptyset$ for $k<l-1$ and $(k, l) \neq(1, r)$; otherwise we can construct a shorter loop. Further, $H_{1} \cap \cdots \cap$ $H_{r}=\emptyset$; otherwise, these elements would be linearly ordered by $\rightarrow \alpha(t)$ for some $t \in \bigcap H_{k}$.

We show that for every $k, H_{k+1}$ is either to the right or to the left of $H_{k}$. Let $k \in[r-2]$ and assume $H_{k+1} \subseteq H_{k}$. Then $H_{k} \cap H_{k+2} \supseteq H_{k+1} \cap H_{k+2} \neq \emptyset$, forcing $k=1$ and $r=k+2=3$; but now also $H_{1} \cap H_{2} \cap H_{3} \neq \emptyset$, a contradiction. A similar contradiction is obtained when assuming $H_{k} \subseteq$ $H_{k+1}$, and also for $H_{r} \subseteq H_{r-1}, H_{r-1} \subseteq H_{r}, H_{1} \subseteq H_{r}$, and $H_{r} \subseteq H_{1}$.

Now assume that $H_{2}$ is to the right of $H_{1}$ (the argument for the other case is similar), then $a_{1}<a_{2}<b_{1}<b_{2}$, see Figure 19 for an illustration.

We proceed by induction. Let $k \in[r-2]$ and assume $H_{k+1}$ is to the right of $H_{k}$, then $a_{k}<$ $a_{k+1}<b_{k}<b_{k+1}$. We show that also $H_{k+2}$ is to the right of $H_{k+1}$. Assume otherwise, then $a_{k+2}<$ $a_{k+1}<b_{k+2}$, hence $a_{k+2}<b_{k}$ and $a_{k}<b_{k+2}$, which implies $H_{k} \cap H_{k+2} \neq \emptyset$, again forcing $k=1$ and $r=k+2=3$ and then a contradiction.

Hence if $H_{2}$ is to the right of $H_{1}$, then the sequence of intervals $H_{1}, \ldots, H_{r}$ proceeds to the right; but the same argument as above then also shows that $H_{1}$ is to the right of $H_{r}$ which is impossible. Similarly, if $H_{2}$ is to the left of $H_{1}$, then the sequence proceeds to the left, and $H_{1}$ then has the impossible task of being to the left of $H_{r}$. Overwhelmed by contradictions, we are forced to accept that $-\rightarrow P$ is irreflexive.

\subsection{Properties of d-path labels}

The main goal of this section is to prove that for every d-path $\alpha$ in $|X|$ there is a track $\rho$ in $X$ with the same labeling and vice versa. First, we show several properties of labels of d-paths. 
Lemma 79. Let $\alpha \in \vec{P}|X|$ and $h: \vec{I} \rightarrow \vec{I}$ a (surjective) reparametrization. Then $\ell(\alpha) \cong \ell(\alpha \circ h)$.

Proof. If $J_{e}^{\alpha}=I_{e, 1}^{\alpha} \cup \cdots \cup I_{e, n_{e}^{\alpha}}^{\alpha}$, then

$$
J_{e}^{\alpha \circ h}=h^{-1}\left(J_{e}^{\alpha}\right)=h^{-1}\left(I_{e, 1}^{\alpha}\right) \cup \cdots \cup h^{-1}\left(I_{e, n_{e}^{\alpha}}^{\alpha}\right)
$$

is a presentation as a union of connected components, so that $n_{e}^{\alpha \circ h}=n_{e}^{\alpha}$ and $I_{e, i}^{\alpha \circ h}=h^{-1}\left(I_{e, i}^{\alpha}\right)$. The result follows from the definition of d-path label.

Lemma 80. Let $f: X \rightarrow Y$ be a map of labeled precubical sets and $e \in E_{Y}$. Then

as a disjoint union.

$$
|f|^{-1}\left(U_{e}^{Y}\right)=\bigsqcup_{e^{\prime} \in E_{f}^{-1}(e)} U_{e^{\prime}}^{X}
$$

Proof. For $p \in|X|$ we have

$$
\begin{aligned}
p \in|f|^{-1}\left(U_{e}^{Y}\right) & \Longleftrightarrow|f|(p) \in U_{e}^{Y} \\
& \Longleftrightarrow e \in \operatorname{ev}(\operatorname{carr}(|f|(p))) \\
& \Longleftrightarrow e \in \operatorname{ev}(f(\operatorname{carr}(p))) \\
& \Longleftrightarrow e \in E_{f}(\operatorname{ev}(\operatorname{carr}(p))) \\
& \Longleftrightarrow \exists e^{\prime} \in E_{f}^{-1}(e): e^{\prime} \in \operatorname{ev}(\operatorname{carr}(p)) \\
& \Longleftrightarrow \exists e^{\prime} \in E_{f}^{-1}(e): p \in U_{e^{\prime}}^{X} .
\end{aligned}
$$

If $p \in U_{e^{\prime}}^{X} \cap U_{e^{\prime \prime}}^{X}$ for $e^{\prime} \neq e^{\prime \prime} \in E_{X}$, then $e^{\prime}, e^{\prime \prime} \in \operatorname{ev}(\operatorname{carr}(p))$. By Lemma 27, $E_{f}\left(e^{\prime}\right), E_{f}\left(e^{\prime \prime}\right) \in$ $\operatorname{ev}(\operatorname{carr}(|f|(p)))$, so $E_{f}\left(e^{\prime}\right) \neq E_{f}\left(e^{\prime \prime}\right)$. Consequently, $e^{\prime}$ and $e^{\prime \prime}$ cannot both belong to $E_{f}^{-1}(e)$.

Lemma 81. For any map of labeled precubical sets $f: X \rightarrow Y$ and $\alpha \in \vec{P}|X|, \ell(\alpha) \cong \ell(|f| \circ \alpha)$.

Proof. By Lemma 80 there is a bijection between connected components of $J_{e}^{|f| \circ \alpha}$ and $\bigcup_{e^{\prime} \in E_{f}^{-1}(e)} J_{e^{\prime}}^{\alpha}$ for every $e \in E_{Y}$. These induce a bijection between the ipomsets $\ell(|f| \circ \alpha)$ and $\ell(\alpha)$. It is easy to check that this is an ipomset isomorphism.

Proposition 82. Let $\alpha, \beta \in \vec{P}|X|$ be such that $\alpha(1)=\beta(0)$. Then $\ell(\alpha * \beta)=\ell(\alpha) * \ell(\beta)$.

Proof. Let $p=\alpha(1)=\beta(0)$ and $l, r:[0,1] \rightarrow[0,1], l(t)=\frac{t}{2}, r(t)=\frac{t+1}{2}$. Then, for each $e \in E_{X}$,

$$
J_{e}^{\alpha * \beta}=\frac{1}{2} J_{e}^{\alpha} \cup\left(\frac{1}{2}\left(J_{e}^{\beta}+\frac{1}{2}\right)=l\left(J_{e}^{\alpha}\right) \cup r\left(J_{e}^{\beta}\right) .\right.
$$

If $e \notin \operatorname{carr}(p)$, then $1 \notin J_{e}^{\alpha}$ and $0 \notin J_{e}^{\beta}$. Thus $l\left(J_{e}^{\alpha}\right)$ and $r\left(J_{e}^{\beta}\right)$ are disjoint, $n_{e}^{\alpha * \beta}=n_{e}^{\alpha}+n_{e}^{\beta}$ and

$$
I_{e, i}^{\alpha * \beta}= \begin{cases}l\left(I_{e, i}^{\alpha}\right) & \text { for } 1 \leq i \leq n_{e}^{\alpha}, \\ r\left(I_{e, i-n_{e}^{\alpha}}^{\beta}\right) & \text { for } n_{e}^{\alpha}<i \leq n_{e}^{\alpha * \beta} .\end{cases}
$$

If $e \in \operatorname{carr}(p)$, then $1 \in J_{e}^{\alpha}$ and $0 \in J_{e}^{\beta}$. Therefore $l\left(J_{e}^{\alpha}\right)$ and $r\left(J_{e}^{\beta}\right)$ are glued along $\frac{1}{2}$ and consequently $n_{e}^{\alpha * \beta}=n_{e}^{\alpha}+n_{e}^{\beta}-1$ and

$$
I_{e, i}^{\alpha * \beta}= \begin{cases}l\left(I_{e, i}^{\alpha}\right) & \text { for } 1 \leq i<n_{e}^{\alpha} \\ l\left(I_{e, n_{e}^{\alpha}}^{\alpha}\right) \cup r\left(I_{e, 1}^{\beta}\right) & \text { for } i=n_{e}^{\alpha} \\ r\left(I_{e, i-n_{e}^{\alpha}}^{\beta}\right) & \text { for } n_{e}^{\alpha}<i<n_{e}^{\alpha * \beta}\end{cases}
$$




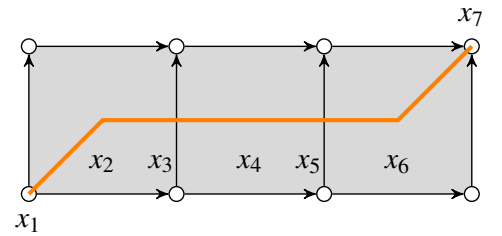

Figure 20. Track $\rho=\left(x_{1}, \ldots, x_{7}\right)$ together with d-path $\alpha$ through center points of $\rho$.

It follows that the maps $i_{e}^{\alpha}: \ell(\alpha) \ni(e, i) \mapsto(e, i) \in \ell(\alpha * \beta)$ and

$$
i_{e}^{\beta}: \ell(\beta) \ni(e, i) \mapsto \begin{cases}\left(e, i+n_{e}^{\alpha}\right) & \text { if } e \notin \operatorname{carr}(p) \\ \left(e, i+n_{e}^{\alpha}-1\right) & \text { if } e \in \operatorname{carr}(p)\end{cases}
$$

glue to the bijection $i: \ell(\alpha) * \ell(\beta) \rightarrow \ell(\alpha * \beta)$. It is elementary to check that $i$ is an ipomset isomorphism.

We record the following easy fact for use in the next proof.

Lemma 83. Let $S$ be a linear pomset and $\alpha \in \vec{P}\left|\square^{S}\right|$ a path such that $\operatorname{carr}(\alpha(t))=\mathbf{y}_{S}$ for some $t \in[0,1]$. Let $x=\operatorname{carr}(\alpha(0))$ and $y=\operatorname{carr}(\alpha(1))$, then $\ell(\alpha)=\ell(x) S_{\ell(y)}$.

Proposition 84. For every d-path $\alpha \in \vec{P}|X|$ there exists a track $\rho: \operatorname{carr}(\alpha(0)) \rightsquigarrow \operatorname{carr}(\alpha(1))$ in $X$ such that $\ell(\alpha) \cong \ell(\rho)$.

Proof. By Lemma 73 there exists a presentation

$$
\alpha=\left(\left(\left|\mathbf{i}_{x_{1}}\right| \circ \beta_{1}\right) *\left(\left|\mathbf{i}_{x_{2}}\right| \circ \beta_{2}\right) * \cdots *\left(\left|\mathbf{i}_{x_{m}}\right| \circ \beta_{m}\right)\right) \circ h
$$

such that $x_{i} \in X_{n_{i}}, \beta_{i} \in \vec{P}\left|\square^{\ell\left(x_{i}\right)}\right|$ and $\mathbf{i}_{x_{i}}: \square^{\ell\left(x_{i}\right)} \rightarrow X$ is the unique HDA map sending $\mathbf{y}_{\ell\left(x_{i}\right)}$ into $x_{i}$ (we replace $\square^{n_{i}}$ with $\square^{\ell\left(x_{i}\right)}$ to obtain compatible labelings and make $\mathbf{i}_{x_{i}}$ HDA maps). We have

$$
\begin{aligned}
\ell(\alpha) & =\ell\left(\left(\left(\left|\mathbf{i}_{x_{1}}\right| \circ \beta_{1}\right) * \cdots *\left(\left|\mathbf{i}_{x_{m}}\right| \circ \beta_{m}\right)\right) \circ h\right) \\
& =\ell\left(\left(\left|\mathbf{i}_{x_{1}}\right| \circ \beta_{1}\right) * \cdots *\left(\left|\mathbf{i}_{x_{m}}\right| \circ \beta_{m}\right)\right) \\
& =\ell\left(\left|\mathbf{i}_{x_{1}}\right| \circ \beta_{1}\right) * \cdots * \ell\left(\left|\mathbf{i}_{x_{m}}\right| \circ \beta_{m}\right) \\
& \left.=\ell\left(\beta_{1}\right) * \cdots * \ell\left(\beta_{m}\right)\right) \\
& =\ell\left(\operatorname{carr}\left(\beta_{1}(0)\right)\right) \ell\left(x_{1}\right) \ell\left(\operatorname{carr}\left(\beta_{1}(1)\right)\right) * \cdots * \ell\left(\operatorname{carr}\left(\beta_{m}(0)\right)\right) \ell\left(x_{m}\right) \ell\left(\operatorname{carr}\left(\beta_{m}(1)\right)\right) \\
& =\ell\left(\operatorname{carr}\left(\beta_{1}(0)\right), x_{1}, \operatorname{carr}\left(\beta_{1}(1)\right)\right) * \cdots * \ell\left(\operatorname{carr}\left(\beta_{m}(0)\right), x_{m}, \operatorname{carr}\left(\beta_{m}(1)\right)\right) \\
& =\ell\left(\operatorname{carr}(\alpha(0)), x_{1}, \operatorname{carr}\left(\beta_{1}(1)\right), \ldots, \operatorname{carr}\left(\beta_{m}(0)\right), x_{m}, \operatorname{carr}(\alpha(1))\right),
\end{aligned}
$$

hence we can set $\rho=\left(\operatorname{carr}(\alpha(0)), x_{1}, \operatorname{carr}\left(\beta_{1}(1)\right), \ldots, \operatorname{carr}\left(\beta_{m}(0)\right), x_{m}, \operatorname{carr}(\alpha(1))\right)$.

For the converse result, we construct a d-path through the center points of a given track, see also [11] and Figure 20 for an example.

Proposition 85. For every track $\rho: x \rightsquigarrow y$ in $X$ there is a d-path $\alpha$ with $\operatorname{carr}(\alpha(0))=x$, $\operatorname{carr}(\alpha(1))=y$, and $\ell(\alpha)=\ell(\rho)$.

Proof. If $\rho=(x)$ is a unit track, we can let $\beta \in \vec{P}\left(\vec{I}^{\mathrm{dim} x}\right)$ be the constant d-path $\beta(t)=\left(\frac{1}{2}, \ldots, \frac{1}{2}\right)$ and $\alpha=\left|\mathbf{i}_{x}\right| \circ \beta$. Otherwise, write $\rho=\left(x_{1}, \ldots, x_{m}\right)$ with $m \geq 2$ and let $n_{i}=\operatorname{dim} x_{i}$ for $i \in[m]$. We construct $\alpha$ as a concatenation of d-paths $\alpha_{1} * \cdots * \alpha_{m-1}$. Let $i \in[m-1]$. 
- If $x_{i} \triangleleft^{*} x_{i+1}$, then $x_{i}=\delta_{A}^{0, \ldots, 0} x_{i+1}$ for a unique set $A \subseteq\left[n_{i+1}\right]$. Let $\beta_{i} \in \vec{P}\left(\vec{I}^{n_{i+1}}\right)$ be the d-path

$$
\beta_{i}(t)=\left(t_{1}, \ldots, t_{n_{i+1}}\right), \quad t_{j}= \begin{cases}\frac{1}{2} t & \text { if } j \in A, \\ \frac{1}{2} & \text { if } j \notin A\end{cases}
$$

and $\alpha_{i}=\left|\mathbf{i}_{x_{i+1}}\right| \circ \beta_{i}$. Then $\operatorname{carr}\left(\alpha_{i}(0)\right)=x_{i}$ and $\operatorname{carr}\left(\alpha_{i}(t)\right)=x_{i+1}$ for $0<t \leq 1$.

- If $x_{i} \triangleright^{*} x_{i+1}$, then $x_{i+1}=\delta_{A}^{1, \ldots, 1} x_{i}$ for a unique set $A \subseteq\left[n_{i}\right]$. Let $\beta_{i} \in \vec{P}\left(\vec{I}^{n_{i}}\right)$ be the d-path

$$
\beta_{i}(t)=\left(t_{1}, \ldots, t_{n_{i}}\right), \quad t_{j}= \begin{cases}\frac{1}{2}+\frac{1}{2} t & \text { if } j \in A, \\ \frac{1}{2} & \text { if } j \notin A\end{cases}
$$

and $\alpha_{i}=\left|\mathbf{i}_{x_{i}}\right| \circ \beta_{i}$. Then $\operatorname{carr}\left(\alpha_{i}(t)\right)=x_{i}$ for $0 \leq t<1$ and $\operatorname{carr}\left(\alpha_{i}(1)\right)=x_{i+1}$.

By construction, $\alpha_{i}(1)=\alpha_{i+1}(0)$ for all $i \in[m-1]$, so the concatenation $\alpha=\alpha_{1} * \cdots * \alpha_{m-1}$ exists. Further, this is a representation as in Lemma 73, hence $\ell(\alpha)=\ell(\rho)$ by Proposition 84 .

\section{Languages of Higher-Dimensional Automata}

We define languages of HDAs and discuss some of their properties.

\subsection{Languages}

Using the work in Sections 5 and 6, we can define languages of HDAs in two different ways. The first one is a straight application of van Glabbeek's track-based approach from [42], and the second one uses d-paths through geometric realizations in the spirit of [13].

Definition 86. A track $\rho: x \rightsquigarrow y$ in an $H D A(X, I, F, \lambda)$ is accepting if $x \in I$ and $y \in F$. The track language of $X$ is $L_{t}(X)=\{\lambda(\rho) \in$ iiPoms $\mid \rho$ accepting track in $X\}$.

A d-path $\alpha \in \vec{P}|X|$ is accepting if $\operatorname{carr}(\alpha(0)) \in I$ and $\operatorname{carr}(\alpha(1)) \in F$. The path language of $X$ is $L_{p}(X)=\{\lambda(\alpha) \in$ iiPoms $\mid \alpha$ accepting d-path in $|X|\}$.

Theorem 87. For every HDA $X, L_{t}(X)=L_{p}(X)$.

Proof. Immediate from Propositions 84 and 85.

From now on we write $L(X)=L_{t}(X)=L_{p}(X)$ and call this set simply the language of $X$. It follows immediately from Proposition 56 that languages of HDAs are sets of interval ipomsets:

Proposition 88. For any $H D A X, L(X) \subseteq$ iiPoms.

The following property allows us to reason about languages using maps from objects $\square^{P}$.

Proposition 89. For any HDA $X$ and any interval ipomset $P, P \in L(X)$ iff there is an HDA map $\square^{P} \rightarrow X$.

Proof. For the forward direction, assume $P \in L(X)$, then there exists a track $\rho: x \rightsquigarrow y$ with $x \in I_{X}$, $y \in F_{X}$, and $\lambda(\rho)=P$. The conclusion follows from Lemma 66 .

For the reverse direction, let $g: \square^{P} \rightarrow X$. Then, by Proposition 67, there exists a track $\rho$ : $i_{\square^{P}} \rightsquigarrow f_{\square^{P}}$ such that $\lambda(\rho)=P, g\left(i_{\square^{P}}\right) \in I_{X}$, and $g\left(f_{\square^{P}}\right) \in F_{X}$. Now Proposition 56 implies that $\lambda(f(\rho))=P$. 
Remark 90. Thanks to Proposition 89, the language of an HDA $X$ may alternatively be defined as the set of interval ipomsets $P$ that admit an HDA map $\square^{P} \rightarrow X$. This definition remains valid even if we do not assume event consistency, hence it may be used to introduce languages also of HDA which are not event consistent. We will expand on this in future work.

We finish this section with some properties of languages of HDAs generated by interval ipomsets. The following is immediate from Proposition 67.

Lemma 91. $P \in L\left(\square^{P}\right)$ for every interval ipomset $P$.

Proposition 92. For all interval ipomsets $P$ and $Q, Q \in L\left(\square^{P}\right)$ iff $Q \sqsubseteq P$.

Proof. The backwards direction is immediate from Lemma 63 and Proposition 89: a subsumption map $f: Q \rightarrow P$ gives rise to $\square^{f}: \square^{Q} \rightarrow \square^{P}$, thus $Q \in L\left(\square^{P}\right)$. For the forward direction, let $\rho$ : $i_{\square^{P}} \rightsquigarrow f_{\square^{P}}$ be an accepting track in $\square^{P}$. We show that $\lambda(\rho) \sqsubseteq P$ by induction on the length of $\rho$.

If $\rho=(x)$, then $i_{\square^{P}}=x=f_{\square^{P}}$, which implies that $P={ }_{P} P_{P}=\ell(\rho)$. Otherwise, there is a presentation $\rho=(x, y) * \tau$. Note that $\ell(x) \cong S_{P}$. There are two cases to consider:

- $x \triangleleft^{*} y$. Then $y(p)=\ulcorner$ for $p \in \operatorname{ev}(y)$ and $y(p)=0$ otherwise. Let $Q$ be an interval ipomset with the same elements as $P,<_{Q}=<_{P},-\rightarrow Q_{Q}=-\rightarrow_{P}, \lambda_{Q}=\lambda_{P}, T_{Q}=T_{P}$; the only difference is that $S_{Q}=\operatorname{ev}(y)$. Then $\square^{P}$ and $\square^{Q}$ are naturally isomorphic as labeled precubical sets and $\tau$ can be regarded as an accepting track in $\square^{Q}$. Moreover, $P={ }_{\ell(x)} \ell(y)_{\ell(y)} * Q$. By induction, $\ell(\tau) \sqsubseteq Q$; using Lemma $48, \ell(\rho)=\ell(x, y) * \ell(\tau) \sqsubseteq P$.

- $x \triangleright^{*} y$. Then

$$
y(p)= \begin{cases}0 & \text { for } p \in P \backslash \mathrm{ev}(x), \\ \ulcorner & \text { for } p \in \operatorname{ev}(y), \\ 1 & \text { for } p \in \operatorname{ev}(x) \backslash \mathrm{ev}(y) .\end{cases}
$$

Let $Q$ be the restriction of $P$ to $P \backslash(\operatorname{ev}(x) \backslash \mathrm{ev}(y))$, then the precubical map $\mathbf{j}_{Q \subseteq P}^{1}: \square^{Q} \rightarrow$ $\square^{P}$ is an injection onto $\{x \mid x(p)=1$ for $p \in \operatorname{ev}(x) \backslash \operatorname{ev}(y)\}$. Furthermore, $\tau$ is a track from $\mathbf{j}_{Q \subseteq P}^{1}\left(i_{\square Q}\right)$ to $\mathbf{j}_{Q \subseteq P}^{1}\left(f_{\square Q}\right)$ lying in $\mathbf{j}_{Q \subseteq P}^{1}\left(\square^{Q}\right)$. Thus, $\tau$ lifts uniquely to an accepting track $\tau^{\prime}$ on $\square Q$. By induction hypothesis, $\ell(\tau) \sqsubseteq Q$, and then with Lemma $48, \ell(\rho)=\ell(x, y) * \ell(\tau) \sqsubseteq$ $\ell(x) \ell(x)_{\ell(y)} * Q=P$.

Remark 93. Example 62 shows that the above proposition fails if $P$ is not an interval ipomset: for $P=2+2, P \notin L\left(\square^{P}\right)$. In general, Proposition 88 implies that if $P \notin$ iiPoms, then $P \notin L\left(\square^{P}\right)$. We will get back to this issue in Example 107 below.

\subsection{Languages are Subsumption-Closed}

Because of Proposition 88 we henceforth restrict ourselves to interval ipomsets.

Definition 94. The weak closure of a set $\mathscr{S} \subseteq$ iiPoms is $\mathscr{S} \downarrow=\{Q \in \mathrm{iiPoms} \mid \exists P \in \mathscr{S}: Q \sqsubseteq P\}$.

That is, $\mathscr{S} \downarrow$ is the smallest subsumption-closed superset of $\mathscr{S}$. The set $\mathscr{S}$ is called weak if $\mathscr{S} \downarrow=\mathscr{S}$. 
Theorem 95. For every $H D A X, L(X) \subseteq$ iiPoms is weak.

Proof. This follows from subsumption closedness of $L\left(\square^{P}\right)$, Proposition 92: Choose interval ipomsets $Q \sqsubseteq P$ with $P \in L(X)$. Proposition 89 gives a map $f: \square^{P} \rightarrow X$ and Proposition 92 gives a map $g: \square \bar{Q} \rightarrow \square^{P}$. The composition $f \circ g$ with Proposition 89 again gives the conclusion.

As a partial converse, we will see in Theorem 101 below that any finite subsumption-closed set of interval ipomsets can be generated by an HDA.

Lemma 96. If $f: X \rightarrow Y$ is an HDA map, then $L(X) \subseteq L(Y)$.

Proof. Let $P \in L(X)$, then Proposition 89 gives a map $\square^{P} \rightarrow X$. Composition with $f$ yields a map $\square^{P} \rightarrow Y$, hence $P \in L(Y)$.

For HDAs generated by interval pomsets, Proposition 92 implies the following.

Lemma 97. $L\left(\square^{P}\right)=\{P\} \downarrow$.

\subsection{Languages are Closed under Union}

We now show that languages of HDAs are closed under union (that is, they form filters). To this end, we introduce coproducts of HDAs. First, the coproduct of precubical sets $X$ and $Y$ is $Z=X \sqcup Y$ given by

$$
Z_{n}=X_{n} \sqcup Y_{n}, \quad \delta_{i}^{v}(z)= \begin{cases}\left(\delta_{X}\right)_{i}^{v}(z) & \text { if } z \in X, \\ \left(\delta_{Y}\right)_{i}^{v}(z) & \text { if } z \in Y .\end{cases}
$$

Definition 98. The coproduct of HDAs $\left(X, I_{X}, F_{X}, \lambda_{X}\right)$ and $\left(Y, I_{Y}, F_{Y}, \lambda_{Y}\right)$ is the HDA $X \sqcup Y=$ $\left(X \sqcup Y, I_{X} \cup I_{Y}, F_{X} \cup F_{Y}, \lambda\right)$ with $\lambda(z)=\lambda_{X}(z)$ if $z \in X$ and $\lambda(z)=\lambda_{Y}(z)$ if $z \in Y$.

It can easily be shown that these are in fact the categorical coproducts in the categories of precubical sets and HDAs, respectively. Next we note that subsumption closure of sets of interval ipomsets distributes over union [18]:

Lemma 99. For any subsets $\mathscr{S}_{1}, \mathscr{S}_{2} \subseteq$ iiPoms, $\left(\mathscr{S}_{1} \cup \mathscr{S}_{2}\right) \downarrow=\mathscr{S}_{1} \downarrow \cup \mathscr{S}_{2} \downarrow$.

Theorem 100. For HDAs $X$ and $Y, L(X \sqcup Y)=L(X) \cup L(Y)$.

Proof. By construction of $X \sqcup Y$, any accepting track in $X \sqcup Y$ is an accepting track in $X$ or in $Y$, and vice versa. The result follows with Lemma 99.

Theorem 101. Let $\mathscr{S} \subseteq$ iiPoms be weak and finite. There is an $H D A X$ with $L(X)=\mathscr{S}$.

Proof. Write $\mathscr{S}=\left\{P_{1}, \ldots, P_{n}\right\} \downarrow$ and let $X=\square^{P_{1}} \sqcup \cdots \sqcup \square^{P_{n}}$. By Lemma 97, $L\left(\square \square^{P_{i}}\right)=\left\{P_{i}\right\} \downarrow$ for all $i=1, \ldots, n$, so using Theorem 100, $L(X)=\left\{P_{1}\right\} \downarrow \cup \cdots \cup\left\{P_{n}\right\} \downarrow=\left(P_{1} \cup \cdots P_{n}\right) \downarrow$ by Lemma 99. 

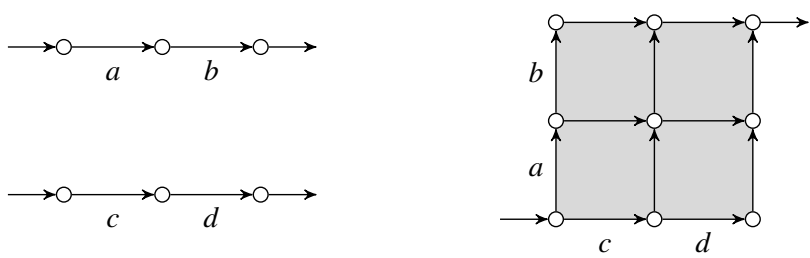

Figure 21. HDAs $X=\square^{(a<b)}$ and $Y=\square^{(c<d)}$ (left) and their tensor product $X \otimes Y$.

\subsection{Languages are Closed under Parallel Composition}

We show below that parallel compositions of HDA languages are languages of tensor products of HDAs. First, the tensor product of precubical sets $X$ and $Y$ is $Z=X \otimes Y$ given by

$$
Z_{n}=\bigsqcup_{k+l=n} X_{k} \times Y_{l}, \quad \delta_{i}^{v}((x, y))= \begin{cases}\left(\delta_{X}\right)_{i}^{v}(x) & \text { if } i \leq \operatorname{dim} x, \\ \left(\delta_{Y}\right)_{i-\operatorname{dim} x}^{v}(y) & \text { if } i>\operatorname{dim} x .\end{cases}
$$

We will below use the important fact that geometric realizations of tensor products are products of geometric realizations [19]:

Lemma 102. For precubical sets $X$ and $Y,|X \otimes Y|=|X| \times|Y|$.

Definition 103. The tensor product of HDAs $\left(X, I_{X}, F_{X}, \lambda_{X}\right)$ and $\left(Y, I_{Y}, F_{Y}, \lambda_{Y}\right)$ is $X \otimes Y=(X \otimes$ $Y, I, F, \lambda)$ with $I=\left\{(x, y) \mid x \in I_{X}, y \in I_{Y}\right\}, F=\left\{(x, y) \mid x \in F_{X}, y \in F_{Y}\right\}$, and $\lambda((x, y))=\lambda_{X}(x) *$ $\lambda_{Y}(y)$.

Above, $\lambda((x, y))=\lambda_{X}(x) * \lambda_{Y}(y)$ denotes the concatenation of $\lambda_{X}(x)$ and $\lambda_{Y}(y)$ as sequences in $! \Sigma$. More formally, one can easily show that $! \Sigma \otimes ! \Sigma=! \Sigma$, so that $\lambda$ is the tensor product of the maps $\lambda_{X}$ and $\lambda_{Y}$.

Remark 104. If $X$ and $Y$ are one-dimensional HDAs, i.e., $X_{2}=Y_{2}=\emptyset$, then $Z=X \otimes Y$ is twodimensional, with $Z_{2}=X_{1} \times Y_{1}$ and $Z_{1}=X_{1} \times Y_{0} \sqcup X_{0} \times Y_{1}$. The labels of 2-cells $(x, y) \in Z_{2}$ are $\lambda((x, y))=\left(\lambda_{X}(x), \lambda_{Y}(y)\right)$, and the labels of 1-cells $(x, y) \in Z_{1}$ are $\lambda(x, y)=\lambda_{X}(x)$ for $x \in X_{1}$ and $\lambda(x, y)=\lambda_{Y}(y)$ for $y \in Y_{1}$. Hence $X \otimes Y$ can be seen as the synchronized product [50, Sec. 2.2.3] of the finite automata $X$ and $Y$.

Lemma 105. For ipomsets $P$ and $Q, \square^{P \| Q} \cong \square^{P} \otimes \square^{Q}$.

Proof. Let $X=\square^{P \| Q}$. As the underlying set of $P \| Q$ is the disjoint union $P \sqcup Q$ and $<_{P \| Q}=$ $<_{P} \sqcup<_{Q}$, any poset map $x:\left(P \| Q,<_{P \| Q}\right) \rightarrow\left\{0,\ulcorner, 1\}\right.$ has a unique decomposition $x=x_{P} \sqcup x_{Q}$ into poset maps $x_{P}:\left(P,<_{P}\right) \rightarrow\left\{0,\ulcorner, 1\}\right.$ and $x_{Q}:\left(Q,<_{Q}\right) \rightarrow\{0,\ulcorner, 1\}$; and any two such maps give rise to a poset map $x$. Hence $X_{n} \cong \bigsqcup_{k+l=n} \square_{k}^{P} \times \square_{l}^{Q}$ as sets. It is easy to see that the face maps agree on both sides, and the same holds for the labeling. For the initial cell we have $i_{\square P \| Q}(p)=\ulcorner$ iff $p \in S_{P \| Q}$ (and 0 otherwise), iff $p \in S_{P}$ or $p \in S_{Q}$, hence $i_{\square^{P} \| Q}$ maps to $i_{\square} \sqcup i_{\square Q}$ under the isomorphism; similarly for the accepting cell.

Definition 106. The parallel composition of subsumption-closed subsets $\mathscr{S}_{1}, \mathscr{S}_{2} \subseteq$ iiPoms is $\mathscr{S}_{1} \| \mathscr{S}_{2}=\left\{P \| Q \mid P \in \mathscr{S}_{1}, Q \in \mathscr{S}_{2}\right\} \downarrow \cap$ iiPoms.

We need to take the intersection with iiPoms above because parallel compositions of interval ipomsets may not be interval. 
Example 107. Let $P$ and $Q$ be the ipomsets $P=(a \longrightarrow b), Q=(c \longrightarrow d)$. Figure 21 shows the one-dimensional HDAs $X=\square^{P}$ and $Y=\square^{Q}$ as well as their tensor product $X \otimes Y=\square^{P \| Q}$ (cf. Example 62 and Figure 14). Now $L(X)=\{P\} \downarrow$ and $L(Y)=\{Q\} \downarrow$, but as $P \| Q$ is not an interval ipomset, $L(X \otimes Y) \neq\{P \| Q\} \downarrow$. Instead,

$$
L(X \otimes Y)=\{P \| Q\} \downarrow \cap \text { iiPoms }=\left\{\left(\begin{array}{l}
a \longrightarrow b \\
c \longrightarrow d
\end{array}\right),\left(\begin{array}{l}
a \longrightarrow b \\
c \longrightarrow d
\end{array}\right)\right\} \downarrow
$$

Theorem 108. For HDAs $X$ and $Y, L(X \otimes Y)=L(X) \| L(Y)$.

Proof. To show $L(X) \| L(Y) \subseteq L(X \otimes Y)$, let $R \in L(X) \| L(Y)$, then there are $P \in L(X)$ and $Q \in$ $L(Y)$ such that $R \sqsubseteq P \| Q$. Let $f: \square^{P} \rightarrow X$ and $g: \square^{Q} \rightarrow Y$ be the maps given by Proposition 89 . There is a composition

$$
\square^{R} \stackrel{(63)}{\longrightarrow} \square^{P \| Q} \stackrel{(105)}{\longrightarrow} \square^{P} \otimes \square^{Q} \stackrel{f \otimes g}{\longrightarrow} X \otimes Y,
$$

thus $R \in L(X \otimes Y)$.

For showing $L(X \otimes Y) \subseteq L(X) \| L(Y)$ we have to do more work. Let $R \in L(X \otimes Y)$, then there is a d-path $\gamma \in \vec{P}|X \otimes Y|$ with $\lambda(\gamma)=R, \operatorname{carr}(\gamma(0)) \in I_{X \otimes Y}$, and $\operatorname{carr}(\gamma(1)) \in F_{X \otimes Y}$. Now $\mid X \otimes$ $Y|=| X|\times| Y \mid$, so let $\alpha$ and $\beta$ be the projections of $\gamma$ to $|X|$ and $|Y|$, respectively. Let $P=\lambda(\alpha)$ and $Q=\lambda(\beta)$. We have $\operatorname{carr}(\alpha(0)) \in I_{X}, \operatorname{carr}(\alpha(1)) \in F_{X}, \operatorname{carr}(\beta(0)) \in I_{Y}$, and $\operatorname{carr}(\beta(1)) \in F_{Y}$, so that $P \in L(X)$ and $Q \in L(Y)$.

We show that $R \sqsubseteq P \| Q$. We have $E_{X \otimes Y}=E_{X} \sqcup E_{Y}$, so for every $e \in E_{X \otimes Y}, J_{e}^{\alpha}=\emptyset$ or $J_{e}^{\beta}=\emptyset$. Further, $J_{e}^{\gamma}=J_{e}^{\alpha} \cup J_{e}^{\beta}$ for every $e \in E_{X \otimes Y}$, so that the presentation $J_{e}^{\gamma}=I_{e, 1}^{\gamma} \cup \cdots \cup I_{e, n_{e}^{\gamma}}^{\gamma}$ is the same as the one for $J_{e}^{\alpha}$ or $J_{e}^{\beta}$. Hence the underlying sets $R=P \sqcup Q$, and $x<_{P} y$ or $x<_{Q} y$ imply $x<_{R} y$.

Regarding the event orders, we work directly with the elementary relations $-\rightarrow \gamma(t)$. Assume $x \rightarrow \gamma(t) y$, then $t \in I_{x}^{\gamma} \cap I_{y}^{\gamma}$. Now, writing $\mathscr{J}^{\alpha}=\left\{J_{e}^{\alpha} \mid e \in E_{X}\right\}$ and $\mathscr{J}^{\beta}=\left\{J_{e}^{\beta} \mid e \in E_{Y}\right\}$,

- if $I_{x}^{\gamma}, I_{y}^{\gamma} \in \mathscr{J}^{\alpha}$, then $x \rightarrow \alpha(t) y$;

- if $I_{x}^{\gamma}, I_{y}^{\gamma} \in \mathscr{J}^{\beta}$, then $x \rightarrow \beta(t) y$;

- if $I_{x}^{\gamma} \in \mathscr{J}^{\alpha}$ and $I_{y}^{\gamma} \in \mathscr{J}^{\beta}$, then $x \in P$ and $y \in Q$, hence $x \rightarrow \rightarrow_{x} y Q$; and

- the case $I_{x}^{\gamma} \in \mathscr{J}^{\beta}$ and $I_{y}^{\gamma} \in \mathscr{J}^{\alpha}$ cannot occur: this would imply $x \in Q$ and $y \in P$ and hence $y \rightarrow-\gamma(t) x$ instead of $x \rightarrow-\gamma(t) y$.

We have shown that $x \rightarrow-\gamma_{(t)} y$ implies $x \rightarrow \rightarrow_{P} \| Q$, so this also holds for the transitive closure $-\rightarrow R$.

\subsection{Language Equivalence is Implied by Bisimulation}

As a final sanity check of our notion of language, we show that bisimilarity of HDAs implies their language equivalence. Fahrenberg [10] has introduced a notion of $h d$-bisimilarity for HDAs which in our setting can be stated as follows. An hd-bisimulation between HDAs $X$ and $Y$ is a graded set $R=\bigcup R_{n}$ with $R_{n} \subseteq X_{n} \times Y_{n}$ such that

(1) $R$ is closed under face maps: for all $(x, y) \in R_{n}, i \in[n]$ and $v \in\{0,1\},\left(\delta_{i}^{v} x, \delta_{i}^{v} y\right) \in R_{n-1}$;

(2) $R$ respects labels: for all $(x, y) \in R, \lambda_{X}(x)=\lambda_{Y}(y)$;

(3) the restrictions $R \cap I_{X} \times I_{Y}$ and $R \cap F_{X} \times F_{Y}$ are bijections;

(4) for all $(x, y) \in R$ and any $x^{\prime} \in X$ and $k \in\left[\operatorname{dim} x^{\prime}\right]$ such that $x=\delta_{k}^{0} x^{\prime}$, there exists $y^{\prime} \in Y$ such that $y=\delta_{k}^{0} y^{\prime}$ and $\left(x^{\prime}, y^{\prime}\right) \in R$; 
(5) for all $(x, y) \in R$ and any $y^{\prime} \in Y$ and $k \in\left[\operatorname{dim} y^{\prime}\right]$ such that $y=\delta_{k}^{0} y^{\prime}$, there exists $x^{\prime} \in X$ such that $x=\delta_{k}^{0} x^{\prime}$ and $\left(x^{\prime}, y^{\prime}\right) \in R$.

Hence initial and accepting cells are related bijectively (3), and (4) whenever a cell in $X$ can be extended, then a related extension is available in $Y$, and vice versa (5). Finally, $X$ and $Y$ are hd-bisimilar if there exists an hd-bisimulation $R \subseteq X \times Y$ : this is an equivalence relation.

As in [10], we can express hd-bisimilarity using open maps [25]. We say that an HDA map $f: X \rightarrow Y$ is open if $f$ is bijective on initial and accepting cells and the following zig-zag property holds for every $x \in X$ : if $y^{\prime} \in Y$ and $k \in\left[\operatorname{dim} y^{\prime}\right]$ are such that $f(x)=\delta_{k}^{0} y^{\prime}$, then there exists $x^{\prime} \in X$ with $x=\delta_{k}^{0} x^{\prime}$ and $y^{\prime}=f\left(x^{\prime}\right)$. The following is shown in [10].

Lemma 109. HDAs $X$ and $Y$ are hd-bisimilar iff there exists an HDA $Z$ and a span of open maps $X \leftarrow Z \rightarrow Y$.

Theorem 110. If HDAs $X$ and $Y$ are hd-bisimilar, then $L(X)=L(Y)$.

Proof. It suffices to assume an open HDA map $f: X \rightarrow Y$; the inclusion $L(X) \subseteq L(Y)$ is then clear by Lemma 96. For the reverse inclusion, let $\sigma=\left(y_{1}, \ldots, y_{m}\right)$ be an accepting track in $Y$. By bijectivity of $f$ on initial cells there is $x_{1} \in I_{X}$ such that $f\left(x_{1}\right)=y_{1}$, and then inductive application of the zig-zag property yields a track $\rho=\left(x_{1}, \ldots, x_{m}\right)$ in $X$ with $f\left(x_{i}\right)=y_{i}$ for all $i$ and $\lambda_{X}(\rho)=$ $\lambda_{Y}(\sigma)$, with $x_{m} \in F_{X}$ because $f$ is bijective on accepting cells.

In [42], van Glabbeek introduces a notion of ST-bisimilarity for HDAs which in our notation is given as follows. An ST-bisimulation between HDAs $X$ and $Y$ is a relation $R$ between tracks in $X$ and $Y$ such that

(1) $R$ is a bijection between initial unit tracks $\left\{(x) \mid x \in I_{X}\right\}$ and $\left\{(y) \mid y \in I_{Y}\right\}$;

(2) $R$ respects accepting cells: for all $(\rho, \sigma) \in R$ such that $\rho: x \rightsquigarrow x^{\prime}$ and $\sigma: y \rightsquigarrow y^{\prime}, x^{\prime} \in F_{X}$ iff $y^{\prime} \in F_{Y}$

(3) $R$ respects labels: for all $(\rho, \sigma) \in R, \ell_{X}(\rho)=\ell_{Y}(\sigma)$;

(4) for all $(\rho, \sigma) \in R$ and track $\rho^{\prime}$ in $X$ such that $\rho$ and $\rho^{\prime}$ may be concatenated, there exists a track $\sigma^{\prime}$ in $Y$ such that $\left(\rho * \rho^{\prime}, \sigma * \sigma^{\prime}\right) \in R$;

(5) for all $(\rho, \sigma) \in R$ and track $\sigma^{\prime}$ in $Y$ such that $\sigma$ and $\sigma^{\prime}$ may be concatenated, there exists a track $\rho^{\prime}$ in $X$ such that $\left(\rho * \rho^{\prime}, \sigma * \sigma^{\prime}\right) \in R$.

That is, whenever a track in $X$ can be extended, then a related extension is available in $Y$ and vice versa. Finally, $X$ and $Y$ are ST-bisimilar if there exists an ST-bisimulation $R$ between them; this is an equivalence relation.

Theorem 111. If HDAs $X$ and $Y$ are ST-bisimilar, then $L(X)=L(Y)$.

Proof. By symmetry it suffices to show the inclusion $L(X) \subseteq L(Y)$. Let $P \in L(X)$, then there is a track $\rho: x \rightsquigarrow x^{\prime}$ in $X$ with $x \in I_{X}, x^{\prime} \in F_{X}$ and $\ell(\rho)=P$. By (1), there is $y \in I_{Y}$ such that the unit tracks $((x),(y)) \in R$. Now $\rho=(x) * \rho$, so using (4) there exists a track $\sigma: y \rightsquigarrow y^{\prime}$ in $Y$ such that $(\rho, \sigma) \in R$, but then by (2), $y^{\prime} \in F_{Y}$. Hence $\sigma$ is an accepting track in $Y$, and by (3), $\ell(\sigma)=\ell(\rho)=$ $P$, so that $P \in L(Y)$.

In [42], other notions of history-preserving and hereditary history-preserving bisimilarity for HDAs are introduced; both imply ST-bisimilarity and, thus, language equivalence. 


\section{References}

[1] Steve Awodey. A cubical model of homotopy type theory. Ann. Pure Appl. Log., 169(12):1270-1294, 2018.

[2] Marek A. Bednarczyk. Categories of Asynchronous Systems. PhD thesis, University of Sussex, UK, 1987.

[3] Marc Bezem, Thierry Coquand, and Simon Huber. A model of type theory in cubical sets. In Ralph Matthes and Aleksy Schubert, editors, TYPES, volume 26 of LIPIcs, pages 107-128. Schloss Dagstuhl - Leibniz-Zentrum für Informatik, 2013.

[4] Marc Bezem, Thierry Coquand, and Simon Huber. The univalence axiom in cubical sets. J. Autom. Reason., 63(2):159-171, 2019.

[5] Zoltán Ésik and Zoltán L. Németh. Higher dimensional automata. J. Autom. Lang. Comb., 9(1):3-29, 2004.

[6] Uli Fahrenberg. Bisimulation for higher-dimensional automata. A geometric interpretation. Research report R-2005-01, Department of Mathematical Sciences, Aalborg University, 2005. Extended version of [10]. https ://www . math . aau . dk/digitalAssets/120/120681_r-2005-01.pdf.

[7] Uli Fahrenberg. Higher-Dimensional Automata from a Topological Viewpoint. PhD thesis, Aalborg University, Denmark, 2005.

[8] Uli Fahrenberg, Christian Johansen, Georg Struth, and Ratan Bahadur Thapa. Generating posets beyond N. In Uli Fahrenberg, Peter Jipsen, and Michael Winter, editors, RAMiCS, volume 12062 of Lect. Notes Comput. Sci., pages 82-99. Springer, 2020. https ://arxiv.org/abs/1910.06162.

[9] Uli Fahrenberg, Christian Johansen, Christopher Trotter, and Krzysztof Ziemianski. Sculptures in concurrency. Log. Methods Comput. Sci., 17(2), 2021. https://lmcs . episciences .org/7363.

[10] Ulrich Fahrenberg. A category of higher-dimensional automata. In Vladimiro Sassone, editor, FoSSaCS, volume 3441 of Lect. Notes Comput. Sci., pages 187-201. Springer, 2005. See also [6].

[11] Lisbeth Fajstrup. Dipaths and dihomotopies in a cubical complex. Adv. Appl. Math., 35(2):188-206, 2005.

[12] Lisbeth Fajstrup, Eric Goubault, Emmanuel Haucourt, Samuel Mimram, and Martin Raussen. Directed Algebraic Topology and Concurrency. Springer, 2016.

[13] Lisbeth Fajstrup, Martin Raussen, and Éric Goubault. Algebraic topology and concurrency. Theor. Comput. Sci., 357(1-3):241-278, 2006.

[14] Jean Fanchon and Rémi Morin. Regular sets of pomsets with autoconcurrency. In Luboš Brim, Petr Jančar, Mojmír Křetínský, and Antonín Kučera, editors, CONCUR, volume 2421 of Lect. Notes Comput. Sci., pages 402-417. Springer, 2002.

[15] Peter C. Fishburn. Intransitive indifference with unequal indifference intervals. J. Math. Psych., 7(1):144-149, 1970.

[16] Peter C. Fishburn. Interval Orders and Interval Graphs: A Study of Partially Ordered Sets. Wiley, 1985.

[17] Jay L. Gischer. The equational theory of pomsets. Theor. Comput. Sci., 61:199-224, 1988.

[18] J. Grabowski. On partial languages. Fund. Inf., 4(2):427, 1981.

[19] Marco Grandis. Directed algebraic topology: models of non-reversible worlds. New mathematical monographs. Cambridge Univ. Press, 2009.

[20] Marco Grandis and Luca Mauri. Cubical sets and their site. Theory Appl. Categ., 11(8):185-211, 2003.

[21] Maurice Herlihy and Jeannette M. Wing. Linearizability: A correctness condition for concurrent objects. ACM Trans. Program. Lang. Syst., 12(3):463-492, 1990.

[22] Ryszard Janicki and Maciej Koutny. Structure of concurrency. Theor. Comput. Sci., 112(1):5-52, 1993.

[23] Ryszard Janicki and Xiang Yin. Modeling concurrency with interval traces. Inf. Comput., 253:78-108, 2017.

[24] Christian Johansen. ST-structures. J. Log. Algebr. Meth. Program., 85(6):1201-1233, 2015. https://arxiv.org/abs/ 1406.0641.

[25] André Joyal, Mogens Nielsen, and Glynn Winskel. Bisimulation from open maps. Inf. Comput., 127(2):164-185, 1996.

[26] Leslie Lamport. The mutual exclusion problem: Part I - A theory of interprocess communication. J. ACM, 33(2):313-326, 1986.

[27] Leslie Lamport. On interprocess communication. Part I: Basic formalism. Distributed Computing, 1(2):77-85, 1986.

[28] Saunders Mac Lane. Categories for the Working Mathematician. Springer, second edition, 1998.

[29] Robin Milner. Communication and Concurrency. Prentice Hall, 1989.

[30] Mogens Nielsen, Gordon D. Plotkin, and Glynn Winskel. Petri nets, event structures and domains, part I. Theor. Comput. Sci., 13:85-108, 1981.

[31] nLab authors. Simplex category. http://ncatlab.org/nlab/show/simplex\%20category, January 2021. Revision 70.

[32] Carl A. Petri. Kommunikation mit Automaten. Number 2 in Schriften des IIM. Institut für Instrumentelle Mathematik, Bonn, 1962.

[33] Vaughan R. Pratt. Modeling concurrency with partial orders. J. Parallel Programming, 15(1):33-71, Feb 1986.

[34] Vaughan R. Pratt. Modeling concurrency with geometry. In POPL, pages 311-322, New York City, 1991. ACM Press.

[35] Vaughan R. Pratt. Chu spaces and their interpretation as concurrent objects. In Jan van Leeuwen, editor, Computer Science Today: Recent Trends and Developments, volume 1000 of Lect. Notes Comput. Sci., pages 392-405. Springer, 1995.

[36] Vaughan R. Pratt. Higher dimensional automata revisited. Math. Struct. Comput. Sci., 10(4):525-548, 2000. 
[37] Vaughan R. Pratt. Transition and cancellation in concurrency and branching time. Math. Struct. Comput. Sci., 13(4):485529, 2003.

[38] Jean-Pierre Serre. Homologie singulière des espaces fibrés. PhD thesis, Ecole Normale Supérieure, Paris, France, 1951.

[39] Mike W. Shields. Concurrent machines. Comput. J., 28(5):449-465, 1985.

[40] Rob J. van Glabbeek. Bisimulations for higher dimensional automata. Email message, 1991. http://theory. stanford.edu/ rvg/hda.

[41] Rob J. van Glabbeek. History preserving process graphs. Unpublished draft, 1996. http://kilby.stanford.edu/ $\sim$ rvg/pub/history.draft.dvi.

[42] Rob J. van Glabbeek. On the expressiveness of higher dimensional automata. Theor. Comput. Sci., 356(3):265-290, 2006. See also [43].

[43] Rob J. van Glabbeek. Erratum to "On the expressiveness of higher dimensional automata". Theor. Comput. Sci., 368(12):168-194, 2006.

[44] Rob J. van Glabbeek and Ursula Goltz. Refinement of actions and equivalence notions for concurrent systems. Acta Inf., 37(4/5):229-327, 2001.

[45] Rob J. van Glabbeek and Gordon D. Plotkin. Configuration structures. In LICS, pages 199-209. IEEE Computer Society, 1995.

[46] Rob J. van Glabbeek and Gordon D. Plotkin. Configuration structures, event structures and Petri nets. Theor. Comput. Sci., 410(41):4111-4159, 2009.

[47] Walter Vogler. Failures semantics based on interval semiwords is a congruence for refinement. Distributed Computing, 4:139-162, 1991.

[48] Walter Vogler. Modular Construction and Partial Order Semantics of Petri Nets, volume 625 of Lecture Notes in Computer Science. Springer, 1992.

[49] Józef Winkowski. An algebraic characterization of the behaviour of non-sequential systems. Inf. Process. Lett., 6(4):105109, 1977.

[50] Glynn Winskel and Mogens Nielsen. Models for concurrency. In Samson Abramsky, Dov M. Gabbay, and Thomas S.E. Maibaum, editors, Handbook of Logic in Computer Science, volume 4. Clarendon Press, Oxford, 1995.

[51] Krzysztof Ziemiański. Spaces of directed paths on pre-cubical sets. Appl. Algebra Eng. Commun. Comput., 28(6):497-525, 2017. https://arxiv.org/abs/1605.08305.

[52] Krzysztof Ziemiański. Spaces of directed paths on pre-cubical sets II. Appl. Comput. Topology, 4:45-78, 2020. https: //arxiv.org/abs/1901.05206. 